\title{
Mesh refinement for uncertainty quantification through model reduction
}

\author{
Jing Li \\ School of Mathematics, University of Minnesota, Minneapolis, MN 55455. Email: \\ lixxx873@umn.edu. \\ Panos Stinis \\ School of Mathematics, University of Minnesota, Minneapolis, MN 55455. Email: \\ stinis@umn.edu.
}

\begin{abstract}
We present a novel way of deciding when and where to refine a mesh in probability space in order to facilitate uncertainty quantification in the presence of discontinuities in random space. A discontinuity in random space makes the application of generalized polynomial chaos expansion techniques prohibitively expensive. The reason is that for discontinuous problems, the expansion converges very slowly. An alternative to using higher terms in the expansion is to divide the random space in smaller elements where a lower degree polynomial is adequate to describe the randomness. In general, the partition of the random space is a dynamic process since some areas of the random space, particularly around the discontinuity, need more refinement than others as time evolves. In the current work we propose a way to decide when and where to refine the random space mesh based on the use of a reduced model. The idea is that a good reduced model can monitor accurately, within a random space element, the cascade of activity to higher degree terms in the chaos expansion. In turn, this facilitates the efficient allocation of computational sources to the areas of random space where they are more needed. For the Kraichnan-Orszag system, the prototypical system to study discontinuities in random space, we present theoretical results which show why the proposed method is sound and numerical results which corroborate the theory.
\end{abstract}

Keywords: Adaptive mesh refinement, Multi-element, gPC, Model reduction.

\section{Introduction}

Generalized polynomial chaos (gPC) is a frequently used approach to represent uncertain quantities when solving differential equations involving uncertainty in initial conditions, boundary conditions, randomness in material 
parameters and etc. Based on the results of Wiener[1], spectral expansion employing Hermite orthogonal polynomials was introduced by Ghanem and Spanos [2] for various uncertainty quantification problems in mechanics. This method was generalized by Xiu and Karniadakis [3,4] to include other families of orthogonal polynomials. When the solution is sufficiently regular with respect to the random inputs, the $\mathrm{gPC}$ expansion has an exponential convergence rate [3]. However, if the solution is not smooth, the rate of convergence of gPC deteriorates similarly to the deterioration of a Fourier expansion of non-smooth functions [5]. The reason for the lack of smoothness can be, for example, the presence of certain values of the random input around which the solution may change qualitatively (this is called a discontinuity in random space). For such problems, the brute force approach of using more terms in the gPC expansion is prohibitively expensive.

An alternative to using higher terms in the expansion is to divide the random space into smaller elements where a lower degree polynomial is adequate to describe the randomness [6]. This approach requires a criterion (a mechanism) to decide how to best partition the random space. Ideally, the criterion will focus on parts of random space, like discontinuities, where there is more sensitive dependence on the value of the random parameters. In addition to the presence of discontinuities in random space, there are problems which simply have too many sources of uncertainty to allow for a high degree expansion in all dimensions of random space. For some problems not all of the sources of uncertainty are equally important. This means that some directions in random space need more refinement than others. So, one needs to be able to identify correctly these directions and allocate accordingly the available computational resources.

In [7], one of the current authors proposed a novel algorithm for performing mesh refinement in physical space by using a reduced model. The algorithm is based on the observation that the need for mesh refinement is dictated by the cascade of energy (or mass depending on the physical context) to scales smaller than the ones resolved. A good reduced model should be able to effect with accuracy the necessary cascade of energy across scales. Thus, a good reduced model can be used to decide when to refine. In [7], a reduced model, called the $t$-model, which was derived through the Mori-Zwanzig formalism [8] was used to monitor the cascade of energy for partial differential equations that develop singularities. This particular model was constructed under the assumption that there is no timescale separation between the resolved and unresolved scales. This absence of timescale separation is an essential feature of problems which require mesh refinement. The solution exhibits small scale features that are important for the dynamic evolution of the solution at all scales.

In the current work we have used the same idea to aid with the task of mesh refinement in random space. In particular, we construct a reduced model for the system resulting from a $\mathrm{gPC}$ expansion of the random solution in each element of the random space (for the problems examined here we have also used the $t$-model as as a reduced model). Then, this reduced model is used to monitor the cascade of energy from the resolved to the unresolved terms in the gPC 
expansion within an element.

What is needed to define a mesh refinement algorithm is a criterion to determine whether it is time to perform mesh refinement. In [7], this criterion was based on monitoring the contribution of memory term from reduced model to the rate of change of the $L_{2}$ norm of the solution at the resolved scales as computed by the reduced model (note that the $L_{2}$ norm corresponds to the mass or energy in many physical contexts). When this contribution value exceeds a prescribed tolerance the algorithm performs mesh refinement. In the current work we use the same criterion to decide if a random element needs to be refined. The suitability of the contribution of memory term to the rate of change of the $L_{2}$ norm as an indicator for the need to refine is shown in Appendix C. In particular, we show that in Kraichnan-Orszag system such contribution for the resolved scales has the same functional form as the expression for the rate of change of the $L_{2}$ error of the reduced model. Thus in this particular system, by keeping, through mesh refinement, the memory term contribution to the rate of change of the $L_{2}$ norm for the resolved scales under a prescribed tolerance, we can keep the error of the calculation under control (see Section 3 for more details).

The paper is organized as follows. In Section 2, we recall the framework for the stochastic Galerkin formulation of a random system. The proposed mesh refinement algorithm is presented in Section 3. Section 4 contains numerical results from the application of the algorithm. Conclusions are drawn in Section 6. Finally, in Appendix A we briefly state the Mori-Zwanzig formalism. Appendix B contains the Galerkin formulation of the Kraichnan-Orszag system as well as the reduced model used in the mesh refinement algorithm. Appendix $\mathrm{C}$ involves a proof of convergence of the reduced model.

\section{2. gPC representation of uncertainty}

Let $(\Omega, \mathcal{A}, \mathcal{P})$ be a probability space, where $\Omega$ is the event space and $\mathcal{P}$ is the probability measure defined on the $\sigma$ - algebra of subsets of $\Omega$. Let $\boldsymbol{\xi}=\left(\xi_{1}, \cdots, \xi_{d}\right)$ be a $d$-dimensional random vector for the random event $\omega \in$ $\Omega$. Without loss of generality, consider an orthonormal generalized polynomial chaos basis $\left\{\Phi_{\mathbf{i}}\right\}_{|\mathbf{i}|=0}^{\infty}$ spanning the space of second-order random processes on this probability space $\left(\mathbf{i}=\left(i_{1}, \cdots, i_{d}\right) \in \mathbb{N}_{0}^{d}\right.$ is a multi-index with $|\mathbf{i}|=i_{1}+\cdots+$ $i_{d}$.) The basis functions $\Phi_{\mathbf{i}}(\boldsymbol{\xi}(\omega))$ are polynomials of degree $|\mathbf{i}|$ with orthonormal relation

$$
\left\langle\Phi_{\mathbf{i}}, \Phi_{\mathbf{j}}\right\rangle=\delta_{\mathbf{i j}},
$$

where $\delta_{\mathbf{i j}}$ is the Kronecker delta and the inner product between two functions $f(\boldsymbol{\xi})$ and $g(\boldsymbol{\xi})$ is defined by

$$
\langle f(\boldsymbol{\xi}), g(\boldsymbol{\xi})\rangle=\int_{\Omega} f(\boldsymbol{\xi}) g(\boldsymbol{\xi}) d \mathcal{P}(\boldsymbol{\xi}) .
$$


A general second-order random process $u(\omega) \in L_{2}(\Omega, \mathcal{A}, \mathcal{P})$ can be expressed by $\mathrm{gPC}$ as

$$
u(\omega)=\sum_{|\mathbf{i}|=0}^{\infty} u_{\mathbf{i}} \Phi_{\mathbf{i}}(\boldsymbol{\xi}(\omega)),
$$

The mean and variance of $u(\omega)$ can be expressed independently of the choice of basis as

$$
\mathbb{E}(u(\boldsymbol{\xi}))=u_{\mathbf{0}}, \quad \operatorname{Var}(u(\boldsymbol{\xi}))=\sum_{|\mathbf{i}|=1}^{\infty} u_{\mathbf{i}}^{2},
$$

respectively. For numerical implementation, (3) is truncated to a finite number of $n$ terms, and we set

$$
u(\omega)=\sum_{|\mathbf{i}|=0}^{p} u_{\mathbf{i}} \Phi_{\mathbf{i}}(\boldsymbol{\xi}(\omega))
$$

where $p$ is the highest total order of the polynomial bases and $n=\left(\begin{array}{c}p \\ d+p\end{array}\right)$.

\section{1. gPC Galerkin method for stochastic differential equations}

Consider the following stochastic differential equation

$$
u_{t}(\mathbf{x}, t ; \omega)=\mathcal{L}(\mathbf{x}, t, \omega ; u)
$$

where $u:=u(\mathbf{x}, t ; \omega)$ is the solution. Operator $\mathcal{L}$ usually involves differentiations in space and can be nonlinear. Appropriate initial conditions and boundary conditions sometimes involving random parameters are assumed. The solution $u$ can be approximated by the truncated gPC expansion

$$
u(\mathbf{x}, t ; \omega)=\sum_{|\mathbf{i}|=0}^{p} \hat{u}_{\mathbf{i}}(\mathbf{x}, t) \Phi_{\mathbf{i}}(\boldsymbol{\xi}(\omega)) .
$$

Substituting equation (7) into the governing system (6), we obtain the following system

$$
\sum_{|\mathbf{i}|=0}^{p} \frac{\partial \hat{u}_{\mathbf{i}}}{\partial t} \Phi_{\mathbf{i}}=\mathcal{L}\left(\mathbf{x}, t, \omega ; \sum_{|\mathbf{i}|=0}^{p} \hat{u}_{\mathbf{i}} \Phi_{\mathbf{i}}\right)
$$

By applying Galerkin projection of (8) onto each element of the orthonormal polynomial basis $\left\{\Phi_{\mathbf{i}}\right\}_{|\mathbf{i}|=0}^{p}$, we derive

$$
\frac{\partial \hat{u}_{\mathbf{i}}}{\partial t}=\left\langle\mathcal{L}\left(\mathbf{x}, t, \omega ; \sum_{|\mathbf{i}|=0}^{p} \hat{u}_{\mathbf{i}} \Phi_{\mathbf{i}}\right), \Phi_{\mathbf{j}}\right\rangle, \quad|\mathbf{j}|=0,1, \cdots, p .
$$

This is a set of $n$ coupled deterministic equations the random modes $\hat{u}_{\mathbf{i}}(\mathbf{x}, t),|\mathbf{i}|=$ $0,1, \cdots, p$. Techniques for deterministic equations can be implemented to solve this system of equations 
For smooth problems, the gPC expansion is efficient due to its exponential convergence. However, for non-smooth problems such as nonlinear problems involving discontinuities in random space, gPC expansions extremely slow. gPC expansions may fail to capture the statistical properties of the solution after a short time [6].

\subsection{Multi-element $g P C$ representation}

An alternative to global gPC representation for efficiently resolving problems with discontinuities in random space is gPC based on a localization of the random space. These localization methods include, among others, multi-element generalized polynomial chaos(ME-gPC) [6], multi-element stochastic collocation $[9,10,11]$, adaptive hierarchical sparse grid collocation [12] and piecewise polynomial multi-wavelets expansion [13, 14, 15].

In this paper, we adopt the ME-gPC approach to deal with discontinuities in random space. Following the notation in [6], we briefly introduce the decomposition of the uniform random space (see [6] for more details). Let $\boldsymbol{\xi}: \Omega \longmapsto \mathbb{R}^{d}$ be a $d$-dimensional random vector defined on the probability space $(\Omega, \mathcal{A}, \mathcal{P})$, where $\xi_{i}, i=1 \cdots, d$ are identical independent distributed(i.i.d) uniform random variables defined as $\xi_{i}: \Omega \longmapsto[-1,1]$ with probability density function(p.d.f) $f_{i}=\frac{1}{2}$. Let $B=[-1,1]^{d} \subset \mathbb{R}^{d}$ be decomposed into $N$ non-overlapping rectangular elements as following:

$$
\begin{aligned}
& B_{k}=\left[a_{1}^{k}, b_{1}^{k}\right) \times\left[a_{2}^{k}, b_{2}^{k}\right) \times \cdots \times\left[a_{d}^{k}, b_{d}^{k}\right], \\
& B=\bigcup_{k=1}^{N} B_{k}, \\
& B_{i} \cap B_{j}=\emptyset \quad \text { if } i \neq j,
\end{aligned}
$$

where $i, j, k=1,2, \cdots, N$. Let $\chi_{k}, k=1,2, \cdots, N$ be the indicator random variables on each of the elements defined by

$$
\chi_{k}= \begin{cases}1 & \text { if } \boldsymbol{\xi} \in B_{k}, \\ 0 & \text { otherwise. }\end{cases}
$$

$\bigcup_{k=1}^{N} \chi_{k}^{-1}(1)$ gives a decomposition of the event space $\Omega$. For each random element, the local random vector is defined by

$$
\zeta^{k}=\left(\zeta_{1}^{k}, \zeta_{2}^{k}, \cdots, \zeta_{d}^{k}\right): \chi_{k}^{-1}(1) \longmapsto B_{k}
$$

subject to the conditional p.d.f

$$
f_{\zeta^{k}}=\frac{1}{2^{d} \operatorname{Prob}\left(\chi_{k}=1\right)}, \quad k=1,2, \cdots, N
$$

where $\operatorname{Prob}\left(\chi_{k}=1\right)=\prod_{i=1}^{d} \frac{b_{i}-a_{i}}{2}$. After that, we transfer each $\boldsymbol{\zeta}^{k}$ to a new random vector defined on $[-1,1]^{d}$ by a map $g_{k}$,

$$
g_{k}\left(\boldsymbol{\zeta}^{k}\right): \zeta_{i}^{k}=\frac{b_{i}^{k}-a_{i}^{k}}{2} \zeta_{i}^{k}+\frac{b_{i}^{k}+a_{i}^{k}}{2}, \quad i=1,2, \cdots, d .
$$


Thus,

$$
\boldsymbol{\xi}^{k}=g_{k}\left(\boldsymbol{\zeta}^{k}\right)=\left(\zeta_{1}^{k}, \zeta_{2}^{k}, \cdots, \zeta_{d}^{k}\right): \chi_{k}^{-1}(1) \longmapsto[-1,1]^{d}
$$

is the new random vector with constant p.d.f $f^{k}=\frac{1}{2^{d}}$. With this decomposition of the random space of $\boldsymbol{\xi}$, we can solve a system of differential equations with random input $\boldsymbol{\xi}$ by combining the local approximations via $\boldsymbol{\zeta}^{k}$ subject to a conditional p.d.f. In practice if the system solution $u(\boldsymbol{\xi})$ is locally approximated by $\hat{u}_{k}\left(\boldsymbol{\zeta}^{k}\right), k=1,2, \cdots, N$, then the $m$ th moment of $u(\boldsymbol{\xi})$ on the entire random space can be obtained by

$$
\begin{aligned}
\mu_{m}(u(\boldsymbol{\xi})) & =\int_{B} u^{m}(\boldsymbol{\xi}) \frac{1}{2^{d}} d \boldsymbol{\xi} \\
& \approx \sum_{k=1}^{N} \operatorname{Prob}\left(\chi_{k}=1\right) \int_{B_{k}} \hat{u}_{k}^{m}\left(\boldsymbol{\zeta}^{k}\right) f_{\boldsymbol{\zeta}^{k}} d \boldsymbol{\zeta}^{k} \\
& =\sum_{k=1}^{N} \operatorname{Prob}\left(\chi_{k}=1\right) \int_{[-1,1]^{d}} \hat{u}_{k}^{m}\left(g_{k}^{-1}\left(\boldsymbol{\xi}^{k}\right)\right) \frac{1}{2^{d}} d \boldsymbol{\xi}^{k}
\end{aligned}
$$

\section{Model reduction and mesh refinement}

This section contains a brief introduction to the main idea behind model reduction and how this can be used to construct a mesh refinement algorithm.

\subsection{Model reduction}

The Galerkin projection of the stochastic system (6) onto the random space transforms it into a deterministic system of coupled equations (9). This deterministic system consists, in general, of partial differential equations (PDEs). After spatial discretization the PDEs are replaced by a system of ordinary differential equations(ODEs). This is our starting point for model reduction. From now on, we call the random coefficients which are the unknown variables of ODEs the random modes.

We split the set of the random modes into two sets, $F$ of resolved modes and $G$ of unresolved ones. Note that this is an internal splitting of the modes of the system. In what follows we always evolve the total set of modes $F \cup G$. The main idea behind model reduction is to construct a modified system for the evolution of the modes in $F$ using the modes in $G$ to effect the necessary transfer of activity between $F$ and $G$.

One can construct a reduced model for the modes in $F$, for example, by using the Mori-Zwanzig formalism [16]. Let $U=\left(\left\{\hat{u}_{\mathbf{i}}\right\}\right), \mathbf{i} \in F \cup G$ be the vector of all PC expansion coefficients. The system of ODEs for their evolution can be written as

$$
\frac{d U(t)}{d t}=R(t, U(t))
$$

where $R(t, U(t))$ is the appropriate right hand side (RHS) after all the necessary discretizations. Let $\hat{U}$ denote the vector of resolved modes and $\tilde{U}$ denote 
the vector of unresolved modes. Similarly, the RHS denoted by $R(t, U)=$ $(\hat{R}(t, U), \tilde{R}(t, U))$. Model reduction constructs a modified system for the evolution of the modes in $\hat{U}$ which should follow accurately these modes without having to solve for the full system. Inevitably, the modified system contains an approximation of the dynamics of the unresolved modes $\tilde{U}$. However, a good reduced model will capture accurately the transfer of activity between the modes in $\hat{U}$ and $\tilde{U}$. It is this property of a good reduced model that we will exploit in constructing our mesh refinement algorithm.

\subsection{The mesh refinement algorithm}

Consider a system of equations with dependence on some random parameters. We decompose the random space in elements as in (10). For each element we consider a system of equations as in (12) resulting from a gPC expansion of the solution within this element. The associated $L_{2}$ norm for the modes in $F$ only is $\hat{E}=\sum_{\mathbf{k} \in F}\left|\hat{u}_{\mathbf{k}}\right|^{2}$. We construct a reduced model for the modes in $F$ which is given by a new system of equations

$$
\frac{d \hat{U}^{\prime}(t)}{d t}=\hat{R}^{\prime}\left(t, \hat{U}^{\prime}(t)\right)
$$

The RHS will be different from the RHS of the equations for $\hat{U}$ in (12). Usually we can divide the RHS into two parts: the Markovian term denoted by $\left.\bar{R}^{\prime}\left(t, \hat{U}^{\prime}(t)\right)\right)$ and the memory term denoted by $\overline{\bar{R}}^{\prime}\left(t, \hat{U}^{\prime}(t)\right)$. In particular, if the reduced model is a t-model, then memory term is the t-model term. The associated $L_{2}$ norm for the modes in $F$ is $\hat{E}^{\prime}=\sum_{\mathbf{k} \in F}\left|\hat{u}_{\mathbf{k}}^{\prime}\right|^{2}$. We can define such an $L_{2}$ norm for each element in the random space. Then $\left|\frac{d \hat{E}^{\prime}}{d t}\right|$ the rate of change of $\hat{E}^{\prime}$ can be compute by the expression $\frac{d \hat{E}^{\prime}}{d t}=2 \sum_{\mathbf{k} \in F} \hat{u}_{\mathbf{k}}^{\prime} \hat{R}^{\prime}\left(t, \hat{U}^{\prime}(t)\right)$. We monitor the contribution of memory term to $\left|\frac{d \hat{E}^{\prime}}{d t}\right|$ that is $\left|2 \sum_{\mathbf{k} \in F} \hat{u}_{\mathbf{k}}^{\prime} \overline{\bar{R}}^{\prime}\left(t, \hat{U}^{\prime}(t)\right)\right|$. For simplicity, we use the following notation

$$
\frac{d \hat{F}^{\prime}}{d t}=2 \sum_{\mathbf{k} \in F} \hat{u}_{\mathbf{k}}^{\prime} \overline{\bar{R}}^{\prime}\left(t, \hat{U}^{\prime}(t)\right)
$$

When $\left|\frac{d \hat{F}^{\prime}}{d t}\right|$ times the probability of the element exceeds a prescribed tolerance we refine the element.

If the random space has $d$ dimensions with $d \geq 2$ then we need to decide not only when and where it is time to refine but also in which direction. Since the modes of the polynomial basis with highest degree contribute most to the transfer of activity from $F$ to $G$, we define $s_{i}=\left|2 \hat{u}_{p_{r} \mathbf{e}_{i}}^{\prime} \overline{\bar{R}}_{p_{r} \mathbf{e}_{i}}^{\prime}\right|$ which is the contribution of the memory term to $\left|\frac{d\left|\hat{u}_{p_{r} \mathbf{e}_{i}}^{\prime}\right|^{2}}{d t}\right|$ with $i=1, \ldots, d . s_{i}$ denotes the contribution of the $i$ th random dimension to $\left|\frac{d \hat{F}^{\prime}}{d t}\right|$. Here, $p_{r} \mathbf{e}_{i}$ is the index vector with the highest degree $p_{r}$ in $i$ th-dimension and degree zero in the rest of the dimensions. 


\section{Mesh refinement algorithm}

Step 1 Choose values $T O L_{1}>0$ and $T O L_{2}>0$ for the tolerances.

Step 2 Mesh refinement:

For time step $t \leftarrow 1, \cdots, N_{T}$

Loop over all elements:

On the $k$-th element $B_{k}$, update the modes for $B_{k}$

If $\left(\left|\frac{d \hat{F}^{\prime}}{d t}\right|\right) \operatorname{Pr}\left(B_{k}\right) \geq T O L_{1}$, loop over all dimensions:

If $s_{i} \geq T O L_{2} \cdot \max _{j=1, \cdots, d} s_{j}$,

split the element $B_{k}$ in two equal parts along the $i$ th dimension and generate local random variables $\xi_{i, 1}$ and $\xi_{i, 2}$ End if

End if

Update the information of the new elements

End loop

We should make a few remarks about the algorithm. First, we do not need to compute the rate of change by numerical differentiation which is inaccurate. Instead, by using the RHS of the equations (13) we obtain an expression for the rate of change which does not involve temporal derivatives (see Appendix B for the relevant expressions for the Kraichnan-Orszag system).

Secondly, for each element we monitor $\left(\left|\frac{d \hat{F}^{\prime}}{d t}\right|\right) \operatorname{Pr}\left(B_{k}\right)$ and not just $\left|\frac{d \hat{F}^{\prime}}{d t}\right|$. This is because each element should be weighted appropriately so that there is no excessive refinement for elements whose contribution is negligible (see (11)).

Thirdly, there are two ways to compute $\left|\frac{d \hat{F}^{\prime}}{d t}\right|$. One way is to evolve, for each element $B_{k}$, both the full and reduced systems of equations (12) and (13). One then uses the values of the modes in $F$ from the reduced system to compute the

expressions involved in $\left|\frac{d \hat{F}^{\prime}}{d t}\right|$. The second way is to evolve only the full system (12) and then use the values of the modes in $F$ to compute the expressions in $\left|\frac{d \hat{F}^{\prime}}{d t}\right|$.

\section{Numerical Examples}

We present results of our mesh refinement algorithm for a simple linear ODE with a random parameter and for the Kraichnan-Orszag three-mode system with random initial conditions.

\subsection{One-dimensional $O D E$}

We begin by considering the simple ODE [6]

$$
\frac{d u}{d t}=-\kappa(\omega) u, \quad u(0 ; \omega)=u_{0}
$$


where $\kappa(\omega) \sim U(-1,1)$. The exact solution of this equation is

$$
u(t, \omega)=u_{0} e^{-\kappa(\omega) t} .
$$

The statistical mean of the solution is

$$
\mu(u(t ; \omega))=\left\{\begin{array}{ll}
\frac{u_{0}}{2 t}\left(e^{t}-e^{-t}\right), & t>0 \\
u_{0}, & t=0
\end{array},\right.
$$

and the variance is

$$
\sigma^{2}(u(t ; \omega))= \begin{cases}\frac{u_{0}^{2}}{4 t}\left(e^{2 t}-e^{-2 t}\right)-\frac{u_{0}^{2}}{4 t^{2}}\left(e^{2 t}+e^{-2 t}-2\right), & t>0 \\ 0, & t=0\end{cases}
$$

Assume

$$
\hat{u}=\sum_{i=0}^{N} \hat{u}_{i} \Phi_{i}(\xi(\omega)), \quad \kappa(\omega)=\sum_{i=0}^{N} \kappa_{i} \Phi(\xi(\omega))
$$

where $\left\{\Phi_{i}\right\}$ are the orthonormal Legendre polynomial chaos bases and $\xi(\omega) \sim$ $U(-1,1)$. The coefficients of the gPC expansion satisfy the ODE system

$$
\frac{\hat{u}_{k}}{d t}=-\sum_{i=0}^{N} \sum_{j=0}^{N} u_{0} \kappa_{i} \hat{u}_{j} e_{i j k}, \quad k=0, \cdots, N
$$

where

$$
e_{i j k}=\int_{\Omega} \Phi_{i}(z) \Phi_{j}(z) \Phi_{k}(z) \rho(z) d z
$$

and $\rho(z)$ is the uniform density in $(-1,1)$. The solution $\hat{u}$ is an approximation of $u$. To implement the adaptive mesh refinement, first we need to construct a reduced model. We have chosen the $t$-model which was originally derived through the Mori-Zwanzig formalism and has been thoroughly studied $[8,17]$. For the system (16), the $t$-model reads

$$
\begin{aligned}
\frac{d \hat{u}_{k}}{d t}= & -u_{0} \sum_{i \in F \cup G} \sum_{j \in F} \kappa_{i} \hat{u}_{j} e_{i j k} \\
& +t u_{0}^{2} \sum_{i \in F \cup G} \sum_{j \in G} \sum_{m \in F \cup G} \sum_{s \in F} \kappa_{i} \kappa_{m} e_{i j k} e_{m s j} \hat{u}_{s}, \quad k \in F
\end{aligned}
$$

where $F=\left\{0,1, \cdots, p_{r}\right\}$ is the set of indices of the resolved modes, and $G=\left\{p_{r}+1, \cdots, p_{f}\right\}$ is the set of indices of the unresolved modes. We study the evolution of the mean and the variance of the solution to the system. There is no discontinuity involved in this problem. However as time increases the lower ordered gPC solution starts to deviate from the exact solution. One way to keep the error under control is to increase the order of the gPC expansion. Another way is to divide the random space into smaller elements so that a lower order expansion is adequate. For our adaptive mesh refinement algorithm we have 
chosen a low order gPC expansion for each element. The price one pays is the need to solve more small systems instead of a large one. Figure 1 shows the curves of the mean(left) and variance(right) via various methods. The results from the refinement algorithm almost reproduce the exact solution while the global gPC solution starts departing from the exact solution as time evolves. We choose the mean and variance of the exact solution as references to study the relative error of each algorithm. We define

$$
\begin{gathered}
\text { Error of mean }=\left|\frac{\mu(u(t ; \omega))-\mu(\hat{u}(t ; \omega))}{\mu(u(t ; \omega))}\right|, \\
\text { Error of variance }=\left|\frac{\sigma^{2}(u(t ; \omega))-\sigma^{2}(\hat{u}(t ; \omega))}{\sigma^{2}(u(t ; \omega))}\right| .
\end{gathered}
$$

In Figure 2, the evolutions of the error of the gPC solution with order 3 and $\mathrm{ME}-\mathrm{gPC}$ are shown for different values of the accuracy control parameters and different orders of the reduced models. The maximum relative errors for the mean and the variance resulting from the use of reduced models of different orders are presented in Table 1. For reasons of comparison we include the relative error from the gPC solution of order 3. As we can see higher ordered reduced models require less elements and at the same time obtain better accuracy. The adaptive meshes at $t=10$ with different accuracy control values $T O L_{1}=10^{-1}$ and $T O L_{1}=10^{-2}$ are demonstrated in Figure 3. The elements on the left end are smaller than those on the right end. This is consistent with the fact that the rate of change of $u(t ; \omega)$ is larger on the left end of the random space. The evolution of the error, of the number of elements and of the error for the variance are shown in Figure 4.
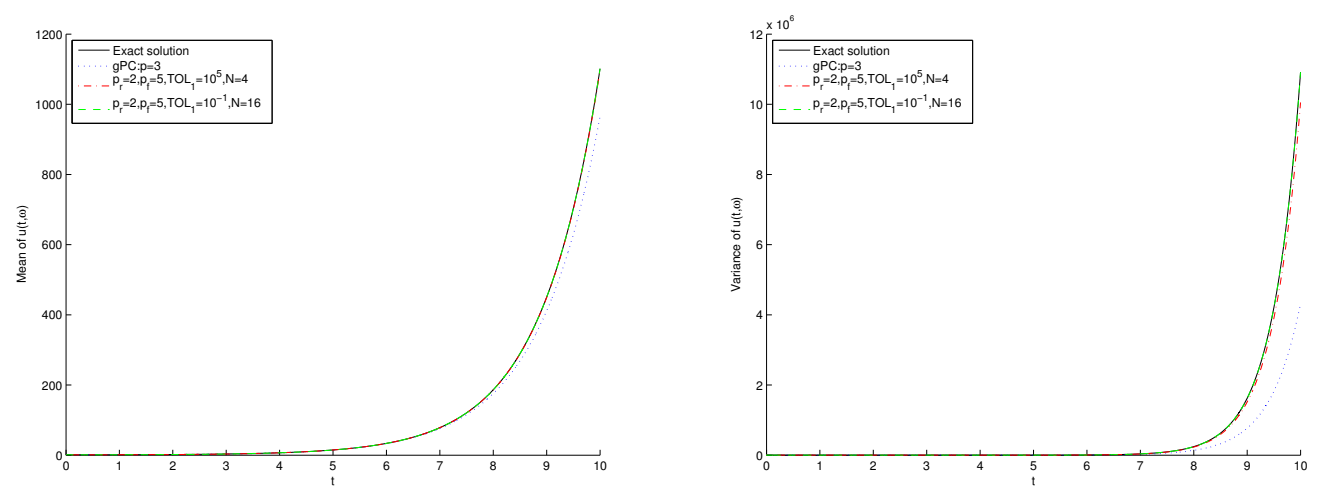

Figure 1: Evolution of mean of $u(t ; \omega)$ (left) and evolution of variance of $u(t ; \omega)$ (right) for the simple ODE.

\subsection{The Kraichnan-Orszag three-mode system}

In [18] it was shown that a Wiener-Hermite expansion does not faithfully represent the dynamics of the system when the random inputs are Gaussian 

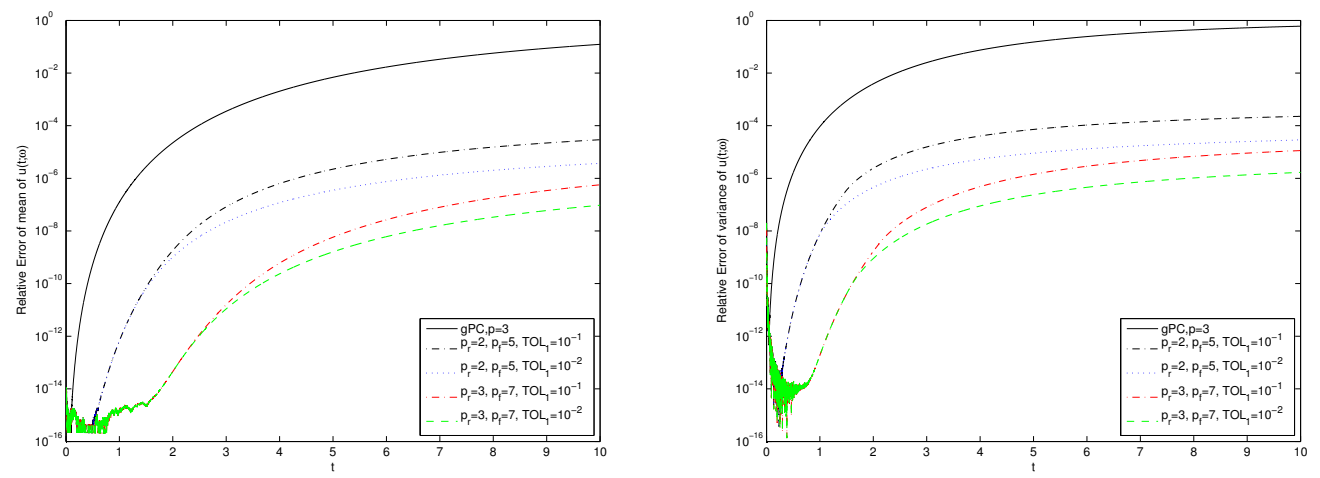

Figure 2: Evolution of relative error of mean of $u(t ; \omega)$ (left) and evolution of relative error of variance of $u(t ; \omega)$ (right) for the simple ODE.

\begin{tabular}{lccc}
\hline & $\mathrm{N}$ & Error of $\mu(u)$ & Error of $\operatorname{var}(u)$ \\
\hline $\mathrm{gPC}, p=5$ & 1 & $3.8 e-3$ & $1.1 e-1$ \\
\hline TOL $L_{1}=10^{-1}$ & & & \\
$p_{r}=2, p_{f}=5$ & 16 & $2.9 e-5$ & $2.3 e-4$ \\
$p_{r}=3, p_{f}=7$ & 9 & $5.7 e-7$ & $1.1 e-5$ \\
\hline TOL $L_{1}=10^{-2}$ & & & \\
$p_{r}=2, p_{f}=5$ & 23 & $3.7 e-6$ & $2.9 e-5$ \\
$p_{r}=3, p_{f}=7$ & 12 & $9.5 e-8$ & $1.7 e-6$ \\
\hline
\end{tabular}

Table 1: Maximum of relative error of mean and variance of solution to the simple ODE when $t \in(0,10]$
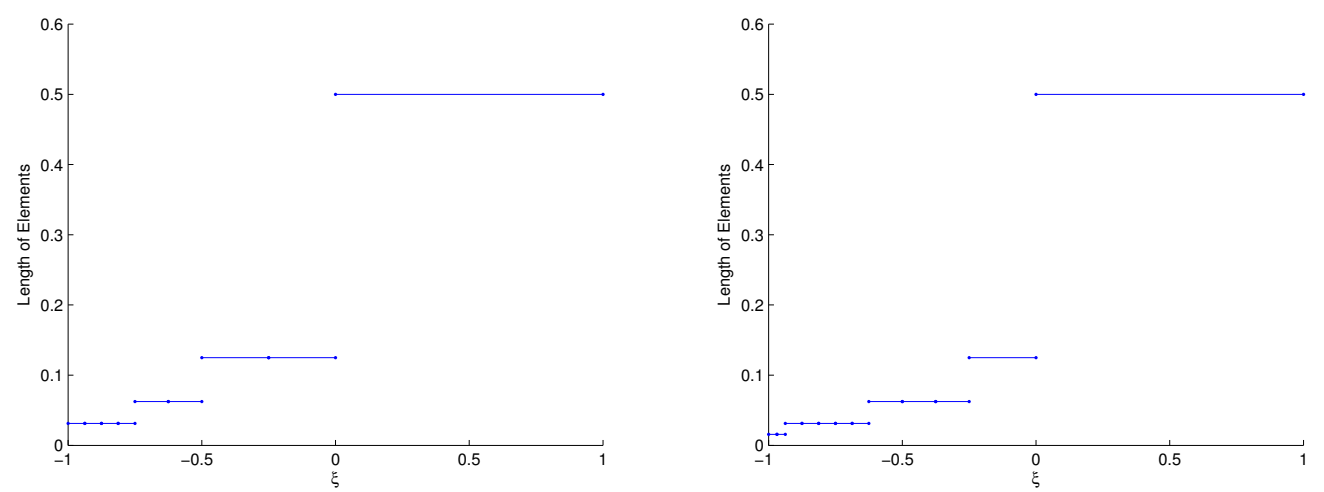

Figure 3: Adaptive meshes for the simple ODE when $p_{r}=3, p_{f}=7, T O L_{1}=10^{-1}$ (left) and TOL $L_{1}=10^{-2}$ (right). 


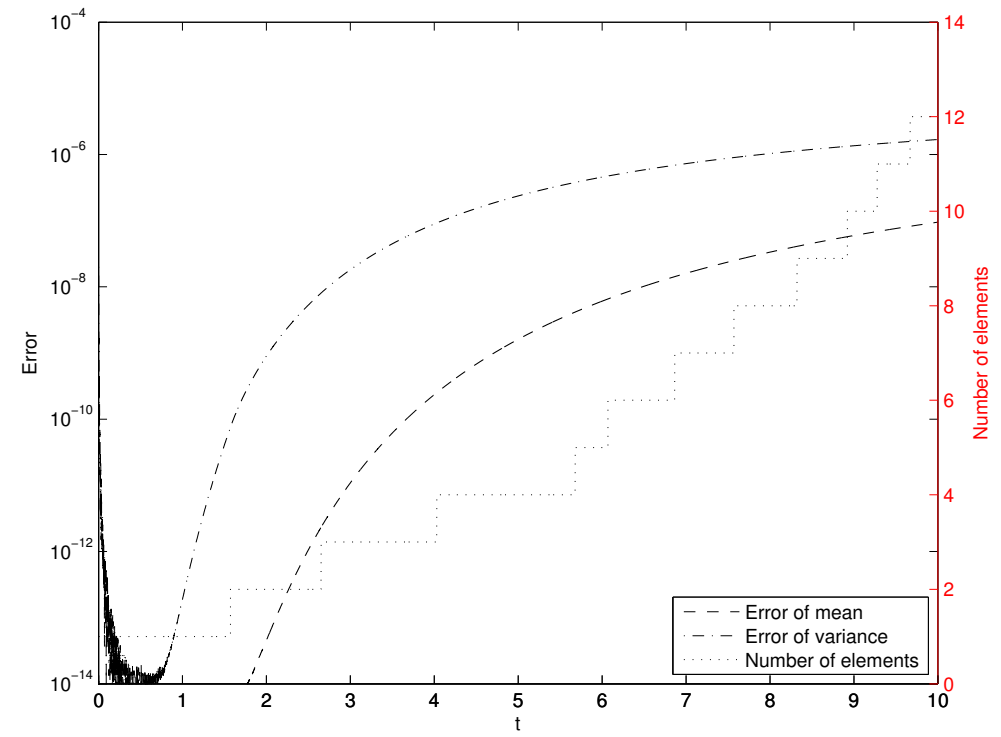

Figure 4: Evolutions of mean error and variance error compared with adaptive meshes for the simple ODE when $p_{r}=3, p_{f}=7, T O L_{1}=10^{-2}$.

variables. A mesh refinement algorithm can efficiently quantify the uncertainty of the system when the random inputs are uniform random variables [6]. For computational convenience, we consider the following system obtained by a linear transformation performed on the original Kraichnan-Orszag three-mode system.

$$
\begin{aligned}
& \frac{d y_{1}}{d t}=y_{1} y_{3}, \\
& \frac{d y_{2}}{d t}=-y_{2} y_{3}, \\
& \frac{d y_{3}}{d t}=-y_{1}^{2}+y_{3}^{2},
\end{aligned}
$$

with the initial conditions

$$
y_{1}(0)=y_{1}(0 ; \omega), \quad y_{2}(0)=y_{2}(0 ; \omega), \quad y_{3}(0)=y_{3}(0 ; \omega),
$$

The discontinuity occurs at the planes $y_{1}=0$ and $y_{2}=0$. Similarly to [6], we consider the case with one random input, two random inputs and three random inputs respectively. Both the Galerkin ODEs and the reduced model of the Kraichnan-Orszag three-mode system are derived in Appendix B. 


\subsubsection{One-dimensional random input}

We choose the initial conditions

$$
y_{1}(0 ; \omega)=1, \quad y_{2}(0 ; \omega)=0.1 \xi(\omega), \quad y_{3}(0 ; \omega)=0,
$$

where $\xi \sim U[-1,1]$. In this case the discontinuity point $y_{2}=0$ is in the random input space.
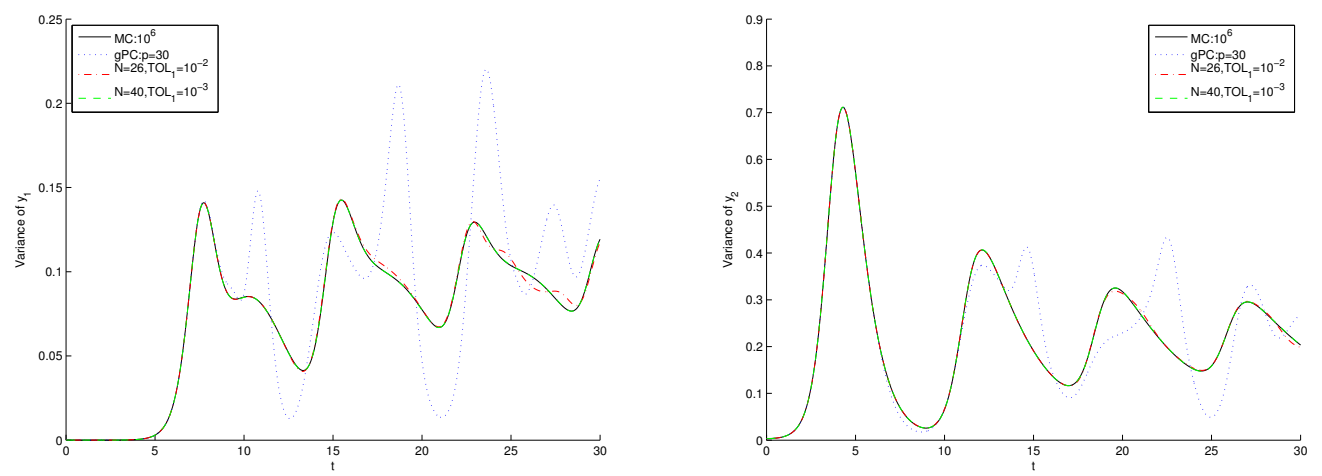

Figure 5: Evolution of the variance of $y_{1}$ (left) and evolution of the variance of $y_{2}$ (right) for the Kraichnan-Orszag three-mode system with 1D random inputs.

We study the variance of each random output $y_{i}, i=1,2,3$ on the time interval $[0,30]$. Figure 5 presents the variance evolution of $y_{1}$ and that of $y_{2}$ estimated by Monte Carlo simulation with 1,000,000 samples and adaptive mesh refinement ME-gPC with polynomial basis order 3 of reduced model and order 7 of full model under various accuracy control values $T O L_{1}$. Failure to capture the properties via the global gPC expansion after a short time is also shown in Figure 5. If we keep the order of the expansion constant and make the tolerance $T O L_{1}$ stricter, more elements are needed by the mesh refinement algorithm and higher accuracy is achieved.

Since ME-gPC achieves higher accuracy than the original gPC on the KraichnanOrszag three-mode system, we use the numerical results of reduced model of order 7 and full model of order 15 and $T O L_{1}=10^{-12}$ as the reference to exam the errors of different sets of reduced model and full model orders (see Table 2 ). For a fixed value of the tolerance $T O L_{1}$, higher order models require fewer elements.

Details of the adaptive meshes from our ME-gPC algorithm around $\xi=0$ are presented in Figure 6. The finest meshes are around the discontinuity of the random space. It demonstrates that our mesh refinement criterion identifies accurately the discontinuity even though the elements are small. Furthermore, when $T O L_{1}$ is extremely small, the meshes exhibit the pattern that the closer the element is to $\xi=0$, the smaller the element. Meanwhile the meshes are symmetric with respect to $\xi=0$ as they should be according to the symmetry of the system. 


\begin{tabular}{lcccc}
\hline & $\mathrm{N}$ & Error of $\operatorname{var}\left(y_{1}\right)$ & Error of $\operatorname{var}\left(y_{2}\right)$ & Error of $\operatorname{var}\left(y_{3}\right)$ \\
\hline$T O L_{1}=10^{-3}$ & & & \\
$p_{r}=3, p_{f}=7$ & 40 & $1.5 e-3$ & $3.0 e-3$ & $9.4 e-4$ \\
$p_{r}=4, p_{f}=9$ & 34 & $5.9 e-3$ & $4.0 e-3$ & $2.2 e-3$ \\
$p_{r}=5, p_{f}=11$ & 32 & $4.2 e-3$ & $4.2 e-3$ & $2.1 e-3$ \\
\hline$T O L_{1}=10^{-6}$ & & & \\
$p_{r}=3, p_{f}=7$ & 110 & $6.0 e-7$ & $5.3 e-6$ & $9.9 e-7$ \\
$p_{r}=4, p_{f}=9$ & 80 & $1.5 e-7$ & $2.9 e-7$ & $1.7 e-7$ \\
$p_{r}=5, p_{f}=11$ & 64 & $1.3 e-7$ & $1.0 e-6$ & $1.4 e-7$ \\
\hline$T O L_{1}=10^{-9}$ & & & & \\
$p_{r}=3, p_{f}=7$ & 256 & $1.0 e-9$ & $8.6 e-9$ & $1.9 e-9$ \\
$p_{r}=4, p_{f}=9$ & 170 & $5.6 e-10$ & $4.6 e-9$ & $8.3 e-10$ \\
$p_{r}=5, p_{f}=11$ & 128 & $1.5 e-10$ & $8.1 e-10$ & $1.1 e-10$ \\
\hline
\end{tabular}

Table 2: Maximum relative errors for the variance of $y_{1}, y_{2}$ and $y_{3}$ when $t \in[0,30]$ for the Kraichnan-Orszag three-mode system with 1D random input

\subsubsection{Two-dimensional random input}

We study the system with initial conditions involving two independent random inputs

$$
y_{1}(0 ; \omega)=1, \quad y_{2}(0 ; \omega)=0.1 \xi_{1}(\omega), \quad y_{3}(0 ; \omega)=\xi_{2}(\omega),
$$

where $\xi_{1}$ and $\xi_{2}$ are independent uniform random variables on $[-1,1]$. In Figure 7 , we plot the evolution of the variance of each random output $y_{i}, i=1,2,3$ subject to a $2 \mathrm{D}$ random input by refinement algorithm with order 4 reduced model and order 7 full model and show the mesh of the random space at time $t=10$ generated by order 4 reduced model and order 7 full model with $T O L_{1}=$ $10^{-4}$ and $T O L_{2}=0.1$. The smallest elements are around the discontinuity $y_{2}=0$ and the results are more sensitive to $\xi_{1}$ because of the discontinuity introduced by $\xi_{1}$. The results for $p_{r}=7$ and $p_{f}=9$ with accuracy control $T O L_{1}=10^{-7}$ and $T O L_{2}=0.1$ are selected to be the references to derive the relative errors to low ordered models. Table 3 presents the relative errors of the variance of $y_{1}, y_{2}$ and $y_{3}$ for different models and different levels of accuracy. We observe similar trends as in the $1 \mathrm{D}$ case, namely that more accurate models require fewer elements if the accuracy tolerance is kept fixed. Also, that stricter accuracy control requires more elements if the order of the models is fixed. To gain the same level of relative errors, the number of the elements increases faster in the $2 \mathrm{D}$ case than the $1 \mathrm{D}$ case.

\subsubsection{Three-dimensional random input}

The initial conditions in this case are

$$
y_{1}(0 ; \omega)=\xi_{1}(\omega), \quad y_{2}(0 ; \omega)=\xi_{2}(\omega), \quad y_{3}(0 ; \omega)=\xi_{3}(\omega),
$$




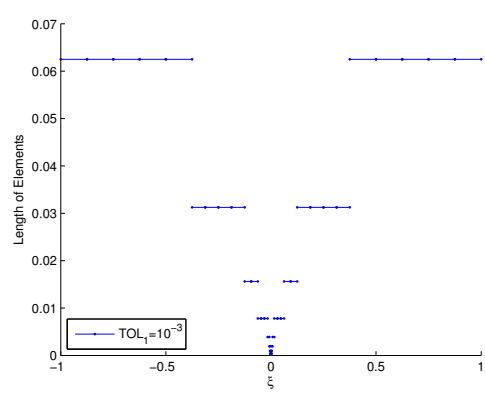

(a)

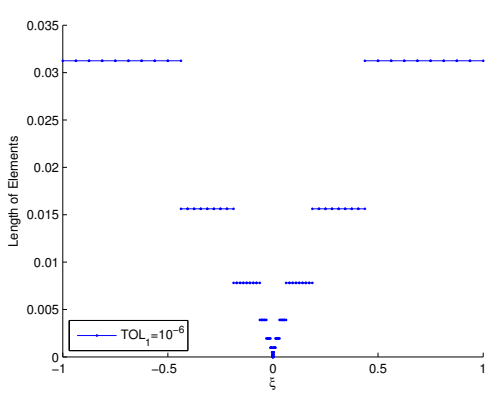

(c)

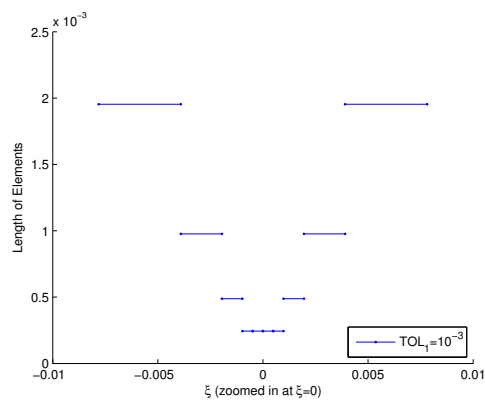

(b)

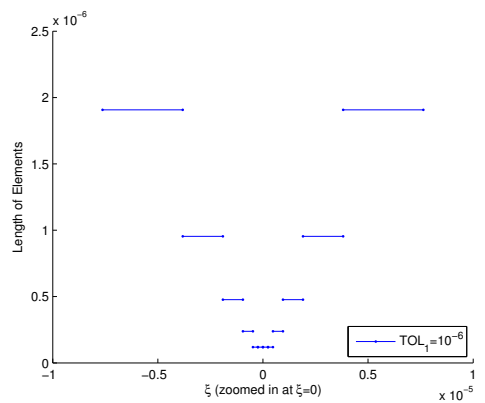

(d)

Figure 6: Adaptive meshes for the Kraichnan-Orszag three-mode system with 1D random input when $p_{r}=3, p_{f}=7$. (a) $T O L_{1}=10^{-3}, N=40$; (b) zoom-in mesh of (a) near $\xi=0$; (c) $T O L_{1}=10^{-6}, N=110$; (d) zoom-in mesh of (c) near $\xi=0$.

where $\xi_{1}$ and $\xi_{2}$ are independent uniform random variables on $[-1,1]$. In this case, discontinuities occur at $y_{1}=0$ and $y_{2}=0$. Figure 8 shows the evolution of variance of $y_{1}$ and $y_{3}$ obtained by order 3 reduced model and order 5 full model with different $T O L_{1}$. The results for a global gPC expansion of order 9 diverges from the Monte Carlo results at $t \approx 3$. On the other had, our ME-gPC algorithms obtains much better results. We choose results from $p_{r}=5, p_{f}=$ $8, T O L_{1}=10^{-5}$ as reference and show the relative errors in Table 4. As we can see, the number of the elements grows dramatically fast in order to gain sufficient accuracy compared to the $1 \mathrm{D}$ and $2 \mathrm{D}$ cases.

\section{Discussion and future work}

We have presented a novel method for adaptive mesh refinement in the context of uncertainty quantification which is based on model reduction. The main idea behind the proposed approach is that a good reduced model can capture accurately the transfer of activity across scales and thus can be utilized to detect when and where higher resolution is needed. We have provided theoretical justification as to why this method is appropriate for adaptive mesh refinement. The 


\begin{tabular}{llccc}
\hline & $\mathrm{N}$ & Error of $\operatorname{var}\left(y_{1}\right)$ & Error of $\operatorname{var}\left(y_{2}\right)$ & Error of $\operatorname{var}\left(y_{3}\right)$ \\
\hline TOL $L_{1}=10^{-1}$ & & & \\
$p_{r}=2, p_{f}=5$ & 20 & $1.2 e-1$ & $2.6 e-1$ & $8.2 e-2$ \\
$p_{r}=3, p_{f}=6$ & 14 & $1.2 e-1$ & $2.1 e-1$ & $1.1 e-1$ \\
$p_{r}=4, p_{f}=7$ & 8 & $2.4 e-2$ & $1.3 e-1$ & $2.4 e-2$ \\
\hline$T O L_{1}=10^{-3}$ & & & \\
$p_{r}=2, p_{f}=5$ & 124 & $2.5 e-4$ & $4.1 e-3$ & $8.2 e-4$ \\
$p_{r}=3, p_{f}=6$ & 106 & $2.9 e-4$ & $6.2 e-3$ & $1.2 e-3$ \\
$p_{r}=4, p_{f}=7$ & 76 & $5.1 e-4$ & $3.4 e-3$ & $1.0 e-3$ \\
\hline TOL $L_{1}=10^{-5}$ & & & & \\
$p_{r}=2, p_{f}=5$ & 554 & $4.1 e-6$ & $1.9 e-4$ & $2.5 e-5$ \\
$p_{r}=3, p_{f}=6$ & 344 & $5.1 e-6$ & $5.2 e-5$ & $1.7 e-5$ \\
$p_{r}=4, p_{f}=7$ & 262 & $9.8 e-6$ & $5.0 e-5$ & $2.3 e-5$ \\
\hline
\end{tabular}

Table 3: The Kraichnan-Orszag three-mode system with 2D random inputs. Maximum relative errors for the variance of $y_{1}, y_{2}$ and $y_{3}$ when $t \in[0,10]$.

\begin{tabular}{lccc}
\hline & $\mathrm{N}$ & Error of $\operatorname{var}\left(y_{1}\right)$ & Error of $\operatorname{var}\left(y_{3}\right)$ \\
\hline$T O L_{1}=10^{-2}$ & & & \\
$p_{r}=2, p_{f}=4$ & 80 & $4.2 e-2$ & $1.1 e-1$ \\
$p_{r}=3, p_{f}=5$ & 48 & $2.3 e-2$ & $5.9 e-2$ \\
$p_{r}=4, p_{f}=6$ & 48 & $1.8 e-2$ & $4.6 e-2$ \\
\hline$T O L_{1}=10^{-3}$ & & & \\
$p_{r}=2, p_{f}=4$ & 368 & $3.3 e-3$ & $8.3 e-2$ \\
$p_{r}=3, p_{f}=5$ & 336 & $2.3 e-3$ & $5.8 e-2$ \\
$p_{r}=4, p_{f}=6$ & 176 & $1.5 e-3$ & $3.9 e-3$ \\
\hline
\end{tabular}

Table 4: The Kraichnan-Orszag three-mode system with 3D random inputs. Maximum relative errors for the variance of $y_{1}, y_{2}$ and $y_{3}$ when $t \in[0,6]$. 


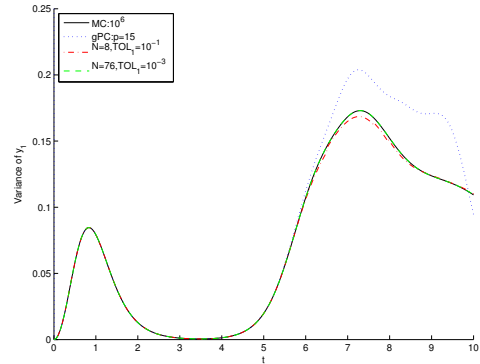

(a)

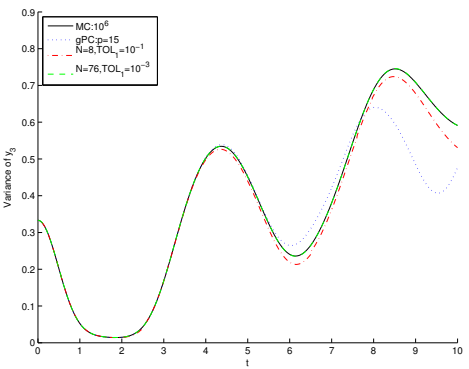

(c)

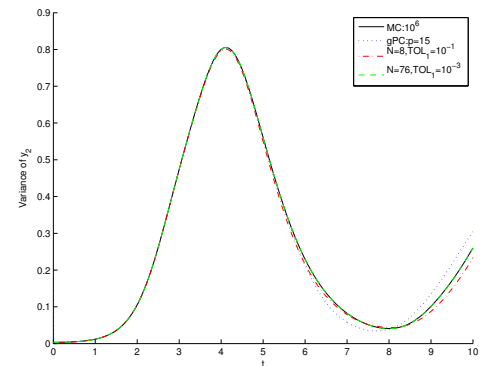

(b)

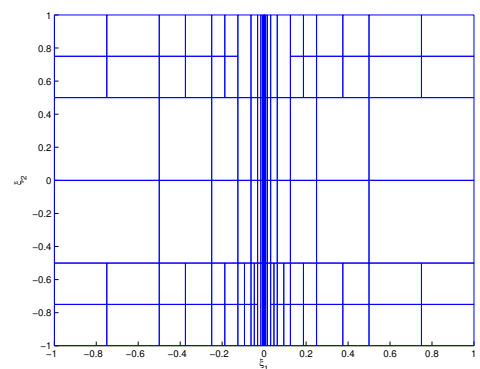

(d)

Figure 7: The Kraichnan-Orszag three-mode system with $2 \mathrm{D}$ random inputs, $p_{r}=4, p_{f}=$ 7, $T O L_{2}=0.1$. (a) Evolution of variance of $y_{1}$; (b) Evolution of variance of $y_{2}$; (c) Evolution of variance of $y_{3}$; (d)Adaptive meshes for $2 \mathrm{D}$ random input when $p_{r}=4, p_{f}=7, T O L_{1}=$ $10^{-4}, N=188$.

proposed approach was implemented in the context of multi-element generalized polynomial chaos expansions. The objective was to perform uncertainty quantification in the presence of discontinuities in the random space. The numerical results for the Kraichnan-Orszag system corroborate the theoretical results.

In its current form the proposed method is applicable to problems where the source of randomness is uniformly distributed. For technical reasons, the method is not applicable in its current form to problems with more elaborate random space distributions, for example Gaussianly distributed randomness. However, as explained in [6], one can treat non-uniform sources of randomness by performing an expansion of the non-uniform randomness in a series of uniform random variables. Such a series expansion is equally applicable for our mesh refinement method and results in this direction will be presented in a future publication. 

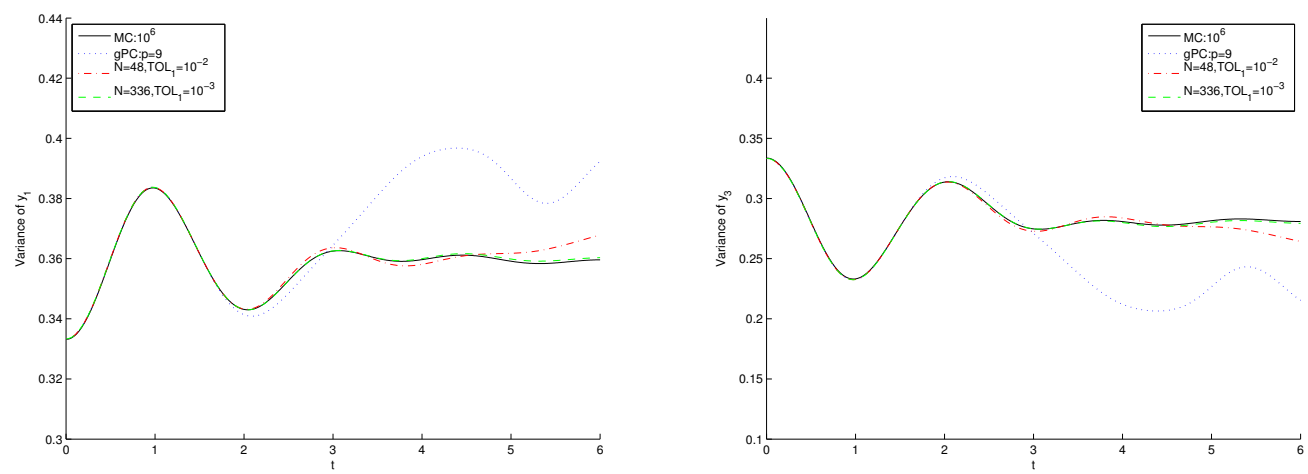

Figure 8: Evolution of the variance of $y_{1}$ (left) and evolution of the variance of $y_{3}$ (right) for the Kraichnan-Orszag three-mode system with 3D random inputs.

\section{Acknowledgements}

This work was supported by the Applied Mathematics Program within the Department of Energy (DOE) Office of Advanced Scientific Computing Research (ASCR) as part of the Collaboratory on Mathematics for Mesoscopic Modeling of Materials (CM4).

\section{References}

[1] N. Wiener, The homogeneous chaos, Amer. J. Math. 60 (1938) 897-936.

[2] R. Ghanem, P. Spanos, Stochastic Finite Elements: a Spectral Approach, Springer-Verlag, 1991.

[3] D. Xiu, G. Karniadakis, The Wiener-Askey polynomial chaos for stochastic differential equations, SIAM J. Sci. Comput. 24 (2) (2002) 619-644.

[4] D. Xiu, Fast Numerical Methods for Stochastic Computations: a Review., Comm. Comput. Phys. 5 (2009) 242-272.

[5] J. Hesthaven, S. Gottlieb, D. Gottlieb, Spectral Methods for TimeDependent Problems, Cambridge University Press, New York, NY, 2007.

[6] X. Wan, G. Karniadakis, An adaptive multi-element generalized polynomial chaos method for stochastic differential equations, J. Comput. Phys. 209 (2) (2005) 617-642.

[7] P. Stinis, A phase transition approach to detecting singularities of PDEs, Comm. App. Math. Comp. Sci. 4 (1) (2009) 217-239.

[8] A. Chorin, O. Hald, R. Kupferman, Optimal prediction with memory, Physica D 166 (2002) 239-257. 
[9] J. Foo, X. Wan, G. Karniadakis, The Multi-element Probabilistic Collocation Method (ME-PCM): Error Analysis and Applications, J. Comput. Phys. 227 (22) (2008) 9572-9595.

[10] J. Foo, G. E. Karniadakis, Multi-element probabilistic collocation method in high dimensions, J. Comput. Phys. 229 (5) (2010) 1536 - 1557.

[11] J. D. Jakeman, A. Narayan, D. Xiu, Minimal multi-element stochastic collocation for uncertainty quantification of discontinuous functions, J. Comput. Phys. 242 (0) (2013) $790-808$.

[12] X. Ma, N. Zabaras, An Adaptive Hierarchical Sparse Grid Collocation Algorithm for the Solution of Stochastic Differential Equations, J. Comput. Phys. 228 (8) (2009) 3084-3113.

[13] O. Le Maitre, O. Knio, H. Najm, R. Ghanem, Uncertainty propagation using Wiener-Haar expansions, J. Comput. Phys. 197 (2004) 28-57.

[14] O. Le Maitre, H. Najm, R. Ghanem, O. Knio, Multi-resolution analysis of Wiener-type uncertainty propagation schemes, J. Comput. Phys. 197 (2004) 502-531.

[15] P. Pettersson, G. Iaccarino, J. Nordström, A stochastic Galerkin method for the Euler equations with Roe variable transformation, Journal of Computational Physics 257, Part A (0) (2014) $481-500$.

[16] A. Chorin, O. Hald, R. Kupferman, Optimal prediction and the MoriZwanzig representation of irreversible processes, PNAS 97 (2000) 29682973.

[17] O. Hald, P. Stinis, Optimal prediction and the rate of decay for solutions of the Euler equations in two and three dimensions, PNAS 104 (16) (2007) $6527-6532$.

[18] S. A. Orszag, L. R. Bissonnette, Dynamical Properties of Truncated Wiener-Hermite Expansions, Physics of Fluids (1958-1988) 10 (12) (1967) 2603-2613.

[19] D. Bernstein, Optimal Prediction of Burgerss' Equation, Multiscale Modeling \& Simulation 6 (1) (2007) 27-52.

\section{Appendix A. The Mori-Zwanzig formalism}

Suppose we are given the full system

$$
\frac{d u(t)}{d t}=R(t, u(t))
$$


where $u=\left(\left\{u_{k}\right\}\right), k \in F \cup G$ with initial condition $u(0)=u_{0}$. The system of ordinary differential equations we are asked to solve can be transformed into a system of linear partial differential equations

$$
\frac{\partial \phi_{k}}{\partial t}=L \phi_{k}, \quad \phi_{k}\left(u_{0}, 0\right)=u_{0 k}, k \in F \cup G
$$

where $L=\sum_{k \in F \cup G} R_{k}\left(u_{0}\right) \frac{\partial}{\partial u_{0 k}}$. The solution of (A.2) is given by $u_{k}\left(u_{0}, t\right)=$ $\phi_{k}\left(u_{0}, t\right)$. Using semigroup notation we can rewrite (A.2) as

$$
\frac{\partial}{\partial t} e^{t L} u_{0 k}=L e^{t L} u_{0 k}
$$

Suppose that the vector of initial conditions can be divided as $u_{0}=\left(\hat{u}_{0}, \tilde{u}_{0}\right)$, where $\hat{u}_{0}$ is the vector of the resolved variables and $\tilde{u}_{0}$ is the vector of the unresolved variables. Let $\mathbb{P}$ be an orthogonal projection on the space of functions of $\hat{u}_{0}$ and $\mathbb{Q}=I-\mathbb{P}$.

Equation (A.2) can be rewritten as

$$
\begin{gathered}
\frac{\partial}{\partial t} e^{t L} u_{0 k}=e^{t L} \mathbb{P} L u_{0 k}+e^{t \mathbb{Q} L} \mathbb{Q} L u_{0 k}+ \\
\int_{0}^{t} e^{(t-s) L} \mathbb{P} L e^{s \mathbb{Q} L} \mathbb{Q} L u_{0 k} d s, k \in F
\end{gathered}
$$

where we have used Dyson's formula

$$
e^{t L}=e^{t \mathbb{Q} L}+\int_{0}^{t} e^{(t-s) L} \mathbb{P} L e^{s \mathbb{Q} L} d s
$$

Equation (A.3) is the Mori-Zwanzig identity. Note that this relation is exact and is an alternative way of writing the original PDE. The first term in (A.3) is usually called Markovian since it depends only on the values of the variables at the current instant, the second is called "noise" and the third "memory". Note that $\mathbb{P} e^{t \mathbb{Q} L} \mathbb{Q} L u_{0 k}=0$ and the operator $e^{t \mathbb{Q} L}$ is called the orthogonal dynamics operator [16].

We can project the Mori-Zwanzig equation (A.3) and find

$$
\frac{\partial}{\partial t} \mathbb{P} e^{t L} u_{0 k}=\mathbb{P} e^{t L} \mathbb{P} L u_{0 k}+\mathbb{P} \int_{0}^{t} e^{(t-s) L} \mathbb{P} L e^{s \mathbb{Q} L} \mathbb{Q} L u_{0 k} d s .
$$

\section{Appendix B. gPC representation and reduced model of the trans- formed Kraichnan-Orszag three-mode system}

We use the truncated gPC expansions to approximate the solution of (17),

$$
\begin{aligned}
& \hat{y}_{1}(t, \xi(\omega))=\sum_{\mathbf{i} \in F \cup G} \hat{y}_{1 \mathbf{i}}(t) \Phi_{\mathbf{i}}(\xi(\omega)), \\
& \hat{y}_{2}(t, \xi(\omega))=\sum_{\mathbf{i} \in F \cup G} \hat{y}_{2 \mathbf{i}}(t) \Phi_{\mathbf{i}}(\xi(\omega)), \\
& \hat{y}_{3}(t, \xi(\omega))=\sum_{\mathbf{i} \in F \cup G} \hat{y}_{3 \mathbf{i}}(t) \Phi_{\mathbf{i}}(\xi(\omega)) .
\end{aligned}
$$


where $F \cup G=\left\{\mathbf{i}: 0 \leq|\mathbf{i}| \leq p_{f}\right\}$, and $F=\left\{\mathbf{i}: 0 \leq|\mathbf{i}| \leq p_{r}\right\}, p_{r}<p_{f}$, $p_{r}, p_{f} \in \mathbb{N}_{0}$. Substitute (B.1) into (17) and perform the Galerkin projection to obtain the system of deterministic ODEs

$$
\begin{aligned}
\frac{d \hat{y}_{1 \mathbf{k}}}{d t} & =\sum_{\mathbf{i} \in F \cup G} \sum_{\mathbf{j} \in F \cup G} \hat{y}_{1 \mathbf{i}} \hat{y}_{3 \mathbf{j}} e_{\mathbf{i} \mathbf{j} \mathbf{k}}, \\
\frac{d \hat{y}_{2 \mathbf{k}}}{d t} & =-\sum_{\mathbf{i} \in F \cup G} \sum_{\mathbf{j} \in F \cup G} \hat{y}_{2 \mathbf{i}} \hat{y}_{3 \mathbf{j}} e_{\mathbf{i} \mathbf{j k}}, \\
\frac{d \hat{y}_{3 \mathbf{k}}}{d t} & =\sum_{\mathbf{i} \in F \cup G} \sum_{\mathbf{j} \in F \cup G}\left(-\hat{y}_{1 \mathbf{i}} \hat{y}_{1 \mathbf{j}}+\hat{y}_{2 \mathbf{i}} \hat{y}_{2 \mathbf{j}}\right) e_{\mathbf{i} \mathbf{j k}}, \quad \mathbf{k} \in F \cup G,
\end{aligned}
$$

where $e_{\mathbf{i j k}}=\int \Phi_{\mathbf{i}} \Phi_{\mathbf{j}} \Phi_{\mathbf{k}} d \mathcal{P}$.

For convenience, we rewrite (B.2) by

$$
\begin{aligned}
& \frac{d \hat{y}_{1 \mathbf{k}}}{d t}=R_{1 \mathbf{k}}, \\
& \frac{d \hat{y}_{2 \mathbf{k}}}{d t}=R_{2 \mathbf{k}}, \\
& \frac{d \hat{y}_{3 \mathbf{k}}}{d t}=R_{3 \mathbf{k}}, \quad \mathbf{k} \in F \cup G
\end{aligned}
$$

where $R_{1 \mathbf{k}}=\sum_{\mathbf{i} \in F \cup G} \sum_{\mathbf{j} \in F \cup G} \hat{y}_{1 \mathbf{i}} \hat{y}_{3 \mathbf{j}} e_{\mathbf{i j k}}, R_{2 \mathbf{k}}=-\sum_{\mathbf{i} \in F \cup G} \sum_{\mathbf{j} \in F \cup G} \hat{y}_{2 \mathbf{i}} \hat{y}_{3 \mathbf{j}} e_{\mathbf{i j k}}$, and $R_{3 \mathbf{k}}=\sum_{\mathbf{i} \in F \cup G} \sum_{\mathbf{j} \in F \cup G}\left(-\hat{y}_{1 \mathbf{i}} \hat{y}_{1 \mathbf{j}}+\hat{y}_{2 \mathbf{i}} \hat{y}_{2 \mathbf{j}}\right) e_{\mathbf{i} \mathbf{j k}}$. We choose one equation from system (B.2),

$$
\frac{d \hat{y}_{1 \mathbf{k}}}{d t}=\sum_{\mathbf{i} \in F \cup G} \sum_{\mathbf{j} \in F \cup G} \hat{y}_{1 \mathbf{i}} \hat{y}_{3 \mathbf{j}} e_{\mathbf{i j k}}
$$

as an example following the Mori-Zwanzig procedure $[8,16,19]$ to derive the $\mathrm{t}$ model. We divide the RHS of (B.4) into two parts: one involving only resolved modes and the residual.

$$
\begin{aligned}
\frac{d \hat{y}_{1 \mathbf{k}}}{d t}= & \left(\sum_{\mathbf{i} \in F} \sum_{\mathbf{j} \in F} \hat{y}_{1 \mathbf{i}} \hat{y}_{3 \mathbf{j}} e_{\mathbf{i j k}}\right) \\
& +\left(\sum_{\mathbf{i} \in G} \sum_{\mathbf{j} \in F} \hat{y}_{1 \mathbf{i}} \hat{y}_{3 \mathbf{j}} e_{\mathbf{i j k}}+\sum_{\mathbf{i} \in F} \sum_{\mathbf{j} \in G} \hat{y}_{1 \mathbf{i}} \hat{y}_{3 \mathbf{j}} e_{\mathbf{i j k}}+\sum_{\mathbf{i} \in G} \sum_{\mathbf{j} \in G} \hat{y}_{1 \mathbf{i}} \hat{y}_{3 \mathbf{j}} e_{\mathbf{i j k}}\right) \\
:= & \bar{R}_{1 \mathbf{k}}+J_{1 \mathbf{k}} .
\end{aligned}
$$

Let $L=\sum_{i=1,2,3} \sum_{\mathbf{k} \in F \cup G} R_{i \mathbf{k}} \frac{\partial}{\partial y_{i \mathbf{k}}}, \mathbb{P}$ be the projection from the full modes to resolved modes and $\mathbb{Q}=I-\mathbb{P}$ is the residual of $\mathbb{P}$. Then we have

$$
\mathbb{Q} L y_{1 \mathbf{k}}=\mathbb{Q} R_{1 \mathbf{k}}=R_{1 \mathbf{k}}-\bar{R}_{1 \mathbf{k}}=J_{1 \mathbf{k}}
$$


A calculation yields

$$
\begin{aligned}
L \mathbb{Q} L y_{1 \mathbf{k}}= & \sum_{\mathbf{i} \in G} \sum_{\mathbf{j} \in F} R_{1 \mathbf{i}} y_{3 \mathbf{j}} e_{\mathbf{i j k}}+\sum_{\mathbf{i} \in G} \sum_{\mathbf{j} \in F} y_{1 \mathbf{i}} R_{3 \mathbf{j}} e_{\mathbf{i j k}} \\
& +\sum_{\mathbf{i} \in F} \sum_{\mathbf{j} \in G} R_{1 \mathbf{i}} y_{3 \mathbf{j}} e_{\mathbf{i j k}}+\sum_{\mathbf{i} \in F} \sum_{\mathbf{j} \in G} y_{1 \mathbf{i}} R_{3 \mathbf{j}} e_{\mathbf{i j k}} \\
& +\sum_{\mathbf{i} \in G} \sum_{\mathbf{j} \in G} R_{1 \mathbf{i}} y_{3 \mathbf{j}} e_{\mathbf{i j k}}+\sum_{\mathbf{i} \in G} \sum_{\mathbf{j} \in G} y_{1 \mathbf{i}} R_{3 \mathbf{j}} e_{\mathbf{i j k}} .
\end{aligned}
$$

Substitute $R_{1 \mathbf{i}}$ and $R_{3 \mathbf{j}}$ into (B.5) and then take the projection $\mathbb{P}$ we have

$$
\mathbb{P} L \mathbb{Q} L y_{1 \mathbf{k}}=\sum_{\mathbf{i} \in F} \sum_{\mathbf{j} \in G} \sum_{\mathbf{s} \in F} \sum_{\mathbf{t} \in F}\left(y_{3 \mathbf{i}} y_{1 \mathbf{s}} y_{3 \mathbf{t}}-y_{1 \mathbf{i}} y_{1 \mathbf{s}} y_{1 \mathbf{t}}+y_{1 \mathbf{i}} y_{2 \mathbf{s}} y_{2 \mathbf{t}}\right) e_{\mathbf{s t j}} e_{\mathbf{i j k}}
$$

$t \mathbb{P} L \mathbb{Q} L y_{1 \mathrm{k}}$ is the t-term in the reduced model and the t-model of (B.4) is derived by

$$
\begin{aligned}
\frac{d \hat{y}_{1 \mathbf{k}}^{\prime}}{d t} & =\sum_{\mathbf{i} \in F} \sum_{\mathbf{j} \in F} \hat{y}_{1 \mathbf{i}}^{\prime} \hat{y}_{3 \mathbf{j}}^{\prime} e_{\mathbf{i j k}} \\
& +t \sum_{\mathbf{i} \in F} \sum_{\mathbf{j} \in G} \sum_{\mathbf{s} \in F} \sum_{\mathbf{t} \in F}\left(\hat{y}_{3 \mathbf{i}}^{\prime} \hat{y}_{1 \mathbf{s}}^{\prime} \hat{y}_{3 \mathbf{t}}^{\prime}-\hat{y}_{1 \mathbf{i}}^{\prime} \hat{y}_{1 \mathbf{s}}^{\prime} \hat{y}_{1 \mathbf{t}}^{\prime}+\hat{y}_{1 \mathbf{i}}^{\prime} \hat{y}_{2 \mathbf{s}}^{\prime} \hat{y}_{2 \mathbf{t}}^{\prime}\right) e_{\mathbf{s t \mathbf { j }}} e_{\mathbf{i} \mathbf{j} \mathbf{k}}
\end{aligned}
$$

For the system (B.2) we choose the $t$-model as the reduced model is given by

$$
\begin{aligned}
\frac{d \hat{y}_{1 \mathbf{k}}^{\prime}}{d t} & =\sum_{\mathbf{i} \in F} \sum_{\mathbf{j} \in F} \hat{y}_{1 \mathbf{i}}^{\prime} \hat{y}_{3 \mathbf{j}}^{\prime} e_{\mathbf{i} \mathbf{j k}} \\
& +t \sum_{\mathbf{i} \in F} \sum_{\mathbf{j} \in G} \sum_{\mathbf{s} \in F} \sum_{\mathbf{t} \in F}\left(\hat{y}_{3 \mathbf{i}}^{\prime} \hat{y}_{1 \mathbf{s}}^{\prime} \hat{y}_{3 \mathbf{t}}^{\prime}-\hat{y}_{1 \mathbf{i}}^{\prime} \hat{y}_{1 \mathbf{s}}^{\prime} \hat{y}_{1 \mathbf{t}}^{\prime}+\hat{y}_{1 \mathbf{i}}^{\prime} \hat{y}_{2 \mathbf{s}}^{\prime} \hat{y}_{2 \mathbf{t}}^{\prime}\right) e_{\mathbf{s t \mathbf { j }}} e_{\mathbf{i j k}} \\
\frac{d \hat{y}_{2 \mathbf{k}}^{\prime}}{d t} & =-\sum_{\mathbf{i} \in F} \sum_{\mathbf{j} \in F} \hat{y}_{2 \mathbf{i}}^{\prime} \hat{y}_{3 \mathbf{j}}^{\prime} e_{\mathbf{i j k}} \\
& +t \sum_{\mathbf{i} \in F} \sum_{\mathbf{j} \in G} \sum_{\mathbf{s} \in F} \sum_{\mathbf{t} \in F}\left(\hat{y}_{3 \mathbf{i}}^{\prime} \hat{y}_{2 \mathbf{s}}^{\prime} \hat{y}_{3 \mathbf{t}}^{\prime}+\hat{y}_{2 \mathbf{i}}^{\prime} \hat{y}_{1 \mathbf{s}}^{\prime} \hat{y}_{1 \mathbf{t}}^{\prime}-\hat{y}_{2 \mathbf{i}}^{\prime} \hat{y}_{2 \mathbf{s}}^{\prime} \hat{y}_{2 \mathbf{t}}^{\prime}\right) e_{\mathbf{s t j}} e_{\mathbf{i} \mathbf{j} \mathbf{k}} \\
\frac{d \hat{y}_{3 \mathbf{k}}^{\prime}}{d t}= & \sum_{\mathbf{i} \in F} \sum_{\mathbf{j} \in F}\left(-\hat{y}_{1 \mathbf{i}}^{\prime} \hat{y}_{1 \mathbf{j}}^{\prime}+\hat{y}_{2 \mathbf{i}}^{\prime} \hat{y}_{2 \mathbf{j}}^{\prime}\right) e_{\mathbf{i} \mathbf{j} \mathbf{k}} \\
& +t \sum_{\mathbf{i} \in F} \sum_{\mathbf{j} \in G} \sum_{\mathbf{s} \in F} \sum_{\mathbf{t} \in F}\left(-2 \hat{y}_{1 \mathbf{i}}^{\prime} \hat{y}_{1 \mathbf{s}}^{\prime} \hat{y}_{3 \mathbf{t}}^{\prime}-2 \hat{y}_{2 \mathbf{i}}^{\prime} \hat{y}_{2 \mathbf{s}}^{\prime} \hat{y}_{2 \mathbf{t}}^{\prime}\right) e_{\mathbf{s t j}} e_{\mathbf{i j k}}
\end{aligned}
$$


For simplicity, we define the following notations:

$$
\begin{aligned}
\hat{R}_{1 \mathbf{k} 1}^{(0)}\left(t, \hat{y}_{1}(t), \hat{y}_{2}(t), \hat{y}_{3}(t)\right)= & \sum_{\mathbf{i} \in F \cup G} \sum_{\mathbf{j} \in F \cup G} \hat{y}_{1 \mathbf{i}} \hat{y}_{3 \mathbf{j}} e_{\mathbf{i j k}} \\
\hat{R}_{1 \mathbf{k} 2}^{(0)}\left(t, \hat{y}_{1}(t), \hat{y}_{2}(t), \hat{y}_{3}(t)\right)= & t \sum_{\mathbf{i} \in F \cup G} \sum_{\mathbf{j} \in I} \sum_{\mathbf{s} \in F \cup G} \sum_{\mathbf{t} \in F \cup G}\left(\hat{y}_{3 \mathbf{i}} \hat{y}_{1 \mathbf{s}} \hat{y}_{3 \mathbf{t}}-\hat{y}_{1 \mathbf{i}} \hat{y}_{1 \mathbf{s}} \hat{y}_{1 \mathbf{t}}\right. \\
& \left.+\hat{y}_{1 \mathbf{i}} \hat{y}_{2 \mathbf{s}} \hat{y}_{2 \mathbf{t}}\right) e_{\mathbf{s t j}} e_{\mathbf{i} \mathbf{j k}} \\
\hat{R}_{2 \mathbf{k} 1}^{(0)}\left(t, \hat{y}_{1}(t), \hat{y}_{2}(t), \hat{y}_{3}(t)\right)= & -\sum_{\mathbf{i} \in F \cup G} \sum_{\mathbf{j} \in F \cup G} \hat{y}_{2 \mathbf{i}} \hat{y}_{3 \mathbf{j}} e_{\mathbf{i j k}} \\
\hat{R}_{2 \mathbf{k} 2}^{(0)}\left(t, \hat{y}_{1}(t), \hat{y}_{2}(t), \hat{y}_{3}(t)\right)= & t \sum_{\mathbf{i} \in F \cup G} \sum_{\mathbf{j} \in I} \sum_{\mathbf{s} \in F \cup G} \sum_{\mathbf{t} \in F \cup G}\left(\hat{y}_{3 \mathbf{i}} \hat{y}_{2 \mathbf{s}} \hat{y}_{3 \mathbf{t}}+\hat{y}_{2 \mathbf{i}} \hat{y}_{1 \mathbf{s}} \hat{y}_{1 \mathbf{t}}\right. \\
& \left.-\hat{y}_{2 \mathbf{i}} \hat{y}_{2 \mathbf{s}} \hat{y}_{2 \mathbf{t}}\right) e_{\mathbf{s t j}} e_{\mathbf{i} \mathbf{j} \mathbf{k}} \\
\hat{R}_{3 \mathbf{i} 1}^{(0)}\left(t, \hat{y}_{1}(t), \hat{y}_{2}(t), \hat{y}_{3}(t)\right)= & \sum_{\mathbf{i} \in F \cup G} \sum_{\mathbf{j} \in F \cup G}\left(-\hat{y}_{1 \mathbf{i}} \hat{y}_{1 \mathbf{j}}+\hat{y}_{2 \mathbf{i}} \hat{y}_{2 \mathbf{j}}\right) e_{\mathbf{i j k}} \\
\hat{R}_{3 \mathbf{k} 2}^{(0)}\left(t, \hat{y}_{1}(t), \hat{y}_{2}(t), \hat{y}_{3}(t)\right)= & t \sum_{\mathbf{i} \in F \cup G} \sum_{\mathbf{j} \in I} \sum_{\mathbf{s} \in F \cup G} \sum_{\mathbf{t} \in F \cup G}\left(-2 \hat{y}_{1 \mathbf{i}} \hat{y}_{1 \mathbf{s}} \hat{y}_{3 \mathbf{t}}\right. \\
& \left.-2 \hat{y}_{2 \mathbf{i}} \hat{y}_{2 \mathbf{s}} \hat{y}_{2 \mathbf{t}}\right) e_{\mathbf{s t j}} e_{\mathbf{i j k}}
\end{aligned}
$$

and

$$
\begin{aligned}
\hat{R}_{1 \mathbf{k} 1}^{(1)}\left(t, \hat{y}_{1}^{\prime}(t), \hat{y}_{2}^{\prime}(t), \hat{y}_{3}^{\prime}(t)\right)= & \sum_{\mathbf{i} \in F} \sum_{\mathbf{j} \in F} \hat{y}_{1 \mathbf{i}}^{\prime} \hat{y}_{3 \mathbf{j}}^{\prime} e_{\mathbf{i j k}} \\
\hat{R}_{1 \mathbf{k} 2}^{(1)}\left(t, \hat{y}_{1}^{\prime}(t), \hat{y}_{2}^{\prime}(t), \hat{y}_{3}^{\prime}(t)\right)= & t \sum_{\mathbf{i} \in F} \sum_{\mathbf{j} \in G} \sum_{\mathbf{s} \in F} \sum_{\mathbf{t} \in F}\left(\hat{y}_{3 \mathbf{i}}^{\prime} \hat{y}_{1 \mathbf{s}}^{\prime} \hat{y}_{3 \mathbf{t}}^{\prime}-\hat{y}_{1 \mathbf{i}}^{\prime} \hat{y}_{1 \mathbf{s}}^{\prime} \hat{y}_{1 \mathbf{t}}^{\prime}\right. \\
& \left.+\hat{y}_{\mathbf{i}}^{\prime} \hat{y}_{2 \mathbf{s}}^{\prime} \hat{y}_{2 \mathbf{t}}^{\prime}\right) e_{\mathbf{s t \mathbf { j }}} e_{\mathbf{i j k}} \\
\hat{R}_{2 \mathbf{k} 1}^{(1)}\left(t, \hat{y}_{1}^{\prime}(t), \hat{y}_{2}^{\prime}(t), \hat{y}_{3}^{\prime}(t)\right)= & -\sum_{\mathbf{i} \in F} \sum_{\mathbf{j} \in F} \hat{y}_{2 \mathbf{i}}^{\prime} \hat{y}_{3 \mathbf{j}}^{\prime} e_{\mathbf{i j k}} \\
\hat{R}_{2 \mathbf{k} 2}^{(1)}\left(t, \hat{y}_{1}^{\prime}(t), \hat{y}_{2}^{\prime}(t), \hat{y}_{3}^{\prime}(t)\right)= & t \sum_{\mathbf{i} \in F} \sum_{\mathbf{j} \in G} \sum_{\mathbf{s} \in F} \sum_{\mathbf{t} \in F}\left(\hat{y}_{3 \mathbf{i}}^{\prime} \hat{y}_{2 \mathbf{s}}^{\prime} \hat{y}_{3 \mathbf{t}}^{\prime}+\hat{y}_{2 \mathbf{i}}^{\prime} \hat{y}_{1 \mathbf{s}}^{\prime} \hat{y}_{1 \mathbf{t}}^{\prime}\right. \\
& \left.-\hat{y}_{2 \mathbf{i}}^{\prime} \hat{y}_{2 \mathbf{s}}^{\prime} \hat{y}_{2 \mathbf{t}}^{\prime}\right) e_{\mathbf{s t \mathbf { j }}} e_{\mathbf{i j k}} \\
\hat{R}_{3 \mathbf{k} 1}^{(1)}\left(t, \hat{y}_{1}^{\prime}(t), \hat{y}_{2}^{\prime}(t), \hat{y}_{3}^{\prime}(t)\right)= & \sum_{\mathbf{i} \in F} \sum_{\mathbf{j} \in F}\left(-\hat{y}_{1 \mathbf{i}}^{\prime} \hat{y}_{1 \mathbf{j}}^{\prime}+\hat{y}_{2 \mathbf{i}}^{\prime} \hat{y}_{2 \mathbf{j}}^{\prime}\right) e_{\mathbf{i j} \mathbf{k}} \\
\hat{R}_{3 \mathbf{k} 2}^{(1)}\left(t, \hat{y}_{1}^{\prime}(t), \hat{y}_{2}^{\prime}(t), \hat{y}_{3}^{\prime}(t)\right)= & t \sum_{\mathbf{i} \in F} \sum_{\mathbf{j} \in G} \sum_{\mathbf{s} \in F} \sum_{\mathbf{t} \in F}\left(-2 \hat{y}_{1 \mathbf{i}}^{\prime} \hat{y}_{1 \mathbf{s}}^{\prime} \hat{y}_{3 \mathbf{t}}^{\prime}\right. \\
& \left.-2 \hat{y}_{2 \mathbf{i}}^{\prime} \hat{y}_{2 \mathbf{s}}^{\prime} \hat{y}_{2 \mathbf{t}}^{\prime}\right) e_{\mathbf{s t \mathbf { j }}} e_{\mathbf{i} \mathbf{j} \mathbf{k}}
\end{aligned}
$$

Note that $R_{i \mathbf{k} j}^{(0)}$ have the same functional form as $R_{i \mathbf{k} j}^{(1)}, i=1,2,3, j=1,2$. Then 
the full model (B.2) and the reduced model (B.7) can be rewritten as

$$
\begin{aligned}
& \frac{d \hat{y}_{1 \mathbf{k}}}{d t}=\sum_{i=1}^{2} a_{1 i}^{(0)} \hat{R}_{1 \mathbf{k} i}^{(0)}\left(t, \hat{y}_{1}, \hat{y}_{2}, \hat{y}_{3}\right), \\
& \frac{d \hat{y}_{2 \mathbf{k}}}{d t}=\sum_{i=1}^{2} a_{2 i}^{(0)} \hat{R}_{2 \mathbf{k} i}^{(0)}\left(t, \hat{y}_{1}, \hat{y}_{2}, \hat{y}_{3}\right), \\
& \frac{d \hat{y}_{3 \mathbf{k}}}{d t}=\sum_{i=1}^{2} a_{3 i}^{(0)} \hat{R}_{3 \mathbf{k} i}^{(0)}\left(t, \hat{y}_{1}, \hat{y}_{2}, \hat{y}_{3}\right), \quad \mathbf{k} \in F \cup G
\end{aligned}
$$

where $a_{11}^{(0)}=1, a_{12}^{(0)}=0, a_{21}^{(0)}=1, a_{22}^{(0)}=0, a_{31}^{(0)}=1, a_{32}^{(0)}=0$, and

$$
\begin{gathered}
\frac{d \hat{y}_{1 \mathbf{k}}^{\prime}}{d t}=\sum_{i=1}^{2} a_{1 i}^{(1)} \hat{R}_{1 \mathbf{k} i}^{(1)}\left(t, \hat{y}_{1}^{\prime}, \hat{y}_{2}^{\prime}, \hat{y}_{3}^{\prime}\right), \\
\frac{d \hat{y}_{2 \mathbf{k}}^{\prime}}{d t}=\sum_{i=1}^{2} a_{2 i}^{(1)} \hat{R}_{2 \mathbf{k} i}^{(1)}\left(t, \hat{y}_{1}^{\prime}, \hat{y}_{2}^{\prime}, \hat{y}_{3}^{\prime}\right), \\
\frac{d \hat{y}_{3 \mathbf{k}}^{\prime}}{d t}=\sum_{i=1}^{2} a_{3 i}^{(1)} \hat{R}_{3 \mathbf{k} i}^{(1)}\left(t, \hat{y}_{1}^{\prime}, \hat{y}_{2}^{\prime}, \hat{y}_{3}^{\prime}\right), \quad \mathbf{k} \in F
\end{gathered}
$$

where $a_{11}^{(1)}=1, a_{12}^{(1)}=1, a_{21}^{(1)}=1, a_{22}^{(1)}=1, a_{31}^{(1)}=1, a_{32}^{(1)}=1$.

The goal of our adaptive mesh refinement approach is to capture the statistical properties of the solution. We have chosen as a criterion for mesh refinement the rate of change of $\hat{E}=\sum_{\mathbf{i} \in F}\left|y_{1 \mathbf{i}}\right|^{2}+\sum_{\mathbf{i} \in F}\left|y_{2 \mathbf{i}}\right|^{2}+\sum_{\mathbf{i} \in F}\left|y_{3 \mathbf{i}}\right|^{2}$.

The rate of change of $\hat{E}$ for the full model is given by

$$
\begin{aligned}
\frac{d \hat{E}}{d t}= & 2 \sum_{\mathbf{k} \in F} \sum_{i=1}^{3} a_{i 1}^{(0)} \operatorname{Re}\left(\hat{R}_{i \mathbf{k} 1}^{(0)}\left(t, \hat{y}_{1}, \hat{y}_{2}, \hat{y}_{3}\right) \hat{y}_{i \mathbf{k}}^{*}\right) \\
& +2 \sum_{\mathbf{k} \in F} \sum_{i=1}^{3} a_{i 2}^{(0)} \operatorname{Re}\left(\hat{R}_{i \mathbf{k} 2}^{(0)}\left(t, \hat{y}_{1}, \hat{y}_{2}, \hat{y}_{3}\right) \hat{y}_{i \mathbf{k}}^{*}\right),
\end{aligned}
$$

where $\left(\hat{y}_{i \mathbf{k}}\right)^{*}$ is the complex conjugate of $\hat{y}_{i \mathbf{k}}$. The rate of change of

$$
\hat{E}^{\prime}=\sum_{\mathbf{i} \in F}\left|y_{1 \mathbf{i}}^{\prime}\right|^{2}+\sum_{\mathbf{i} \in F}\left|y_{2 \mathbf{i}}^{\prime}\right|^{2}+\sum_{\mathbf{i} \in F}\left|y_{3 \mathbf{i}}^{\prime}\right|^{2}
$$

for the reduced model is given by

$$
\begin{aligned}
\frac{d \hat{E}^{\prime}}{d t}= & 2 \sum_{\mathbf{k} \in F} \sum_{i=1}^{3} a_{i 1}^{(1)} \operatorname{Re}\left(\hat{R}_{i \mathbf{k} 1}^{(0)}\left(t, \hat{y}_{1}^{\prime}, \hat{y}_{2}^{\prime}, \hat{y}_{3}^{\prime}\right)\left(\hat{y}_{i \mathbf{k}}^{\prime}\right)^{*}\right) \\
& +2 \sum_{\mathbf{k} \in F} \sum_{i=1}^{3} a_{i 2}^{(0)} \operatorname{Re}\left(\hat{R}_{i \mathbf{k} 2}^{(0)}\left(t, \hat{y}_{1}^{\prime}, \hat{y}_{2}^{\prime}, \hat{y}_{3}^{\prime}\right)\left(\hat{y}_{i \mathbf{k}}^{\prime}\right)^{*}\right),
\end{aligned}
$$

where $\left(\hat{y}_{i \mathbf{k}}^{\prime}\right)^{*}$ is the complex conjugate of $\hat{y}_{i \mathbf{k}}^{\prime}$. 


\section{Appendix C. Error of the $t$-model for the Kraichnan-Orszag three- mode system}

We will show the relation between the rate of change of $\hat{E}^{\prime}=\sum_{\mathbf{i} \in F}\left|y_{1 \mathbf{i}}^{\prime}\right|^{2}+$ $\sum_{\mathbf{i} \in F}\left|y_{2 \mathbf{i}}^{\prime}\right|^{2}+\sum_{\mathbf{i} \in F}\left|y_{3 \mathbf{i}}^{\prime}\right|^{2}=\left\|\hat{\mathbf{y}}^{\prime}\right\|_{L_{2}(\Omega)}^{2}$ and the error of the $t$-model. Use the notations from Appendix. Appendix $\mathrm{B}$, and let $\mathbf{P}$ be the projection onto the space spanned by $\left\{\Phi_{\mathbf{i}} \mid \mathbf{i} \in F\right\}$. Let $\mathbf{B}\left(y_{i}, y_{j}\right)=y_{i} y_{j}, i, j=1,2,3$. Then, the Kraichnan-Orszag three-mode system (17) can be written as

$$
\frac{d y_{1}}{d t}=\mathbf{B}\left(y_{1}, y_{3}\right), \quad \frac{d y_{2}}{d t}=-\mathbf{B}\left(y_{2}, y_{3}\right), \quad \frac{d y_{3}}{d t}=-\mathbf{B}\left(y_{1}, y_{1}\right)+\mathbf{B}\left(y_{2}, y_{2}\right) .
$$

and its projection

$$
\begin{aligned}
\frac{d \mathbf{P} y_{1}}{d t} & =\mathbf{P B}\left(y_{1}, y_{3}\right), \\
\frac{d \mathbf{P} y_{2}}{d t} & =-\mathbf{P B}\left(y_{2}, y_{3}\right), \\
\frac{d \mathbf{P} y_{3}}{d t} & =-\mathbf{P B}\left(y_{1}, y_{1}\right)+\mathbf{P B}\left(y_{2}, y_{2}\right) .
\end{aligned}
$$

The $t$-model can be written as

$$
\begin{aligned}
\frac{d \hat{y}_{1}^{\prime}}{d t}= & \mathbf{P B}\left(\hat{y}_{1}^{\prime}, \hat{y}_{3}^{\prime}\right)+t \mathbf{P}\left\{\mathbf{B}\left((\mathbf{I}-\mathbf{P}) \mathbf{B}\left(\hat{y}_{1}^{\prime}, \hat{y}_{3}^{\prime}\right), \hat{y}_{3}^{\prime}\right)\right\} \\
& +t \mathbf{P}\left\{\mathbf{B}\left(\hat{y}_{1}^{\prime},-(\mathbf{I}-\mathbf{P}) \mathbf{B}\left(\hat{y}_{1}^{\prime}, \hat{y}_{1}^{\prime}\right)+(\mathbf{I}-\mathbf{P}) \mathbf{B}\left(\hat{y}_{2}^{\prime}, \hat{y}_{2}^{\prime}\right)\right)\right\}, \\
\frac{d \hat{y}_{2}^{\prime}}{d t}= & -\mathbf{P B}\left(\hat{y}_{2}^{\prime}, \hat{y}_{3}^{\prime}\right)-t \mathbf{P}\left\{\mathbf{B}\left(-(\mathbf{I}-\mathbf{P}) \mathbf{B}\left(\hat{y}_{2}^{\prime}, \hat{y}_{3}^{\prime}\right), \hat{y}_{3}^{\prime}\right)\right\} \\
& -t \mathbf{P}\left\{\mathbf{B}\left(\hat{y}_{2}^{\prime},-(\mathbf{I}-\mathbf{P}) \mathbf{B}\left(\hat{y}_{1}^{\prime}, \hat{y}_{1}^{\prime}\right)+(\mathbf{I}-\mathbf{P}) \mathbf{B}\left(\hat{y}_{2}^{\prime}, \hat{y}_{2}^{\prime}\right)\right)\right\}, \\
\frac{d \hat{y}_{3}^{\prime}}{d t}= & -\mathbf{P B}\left(\hat{y}_{1}^{\prime}, \hat{y}_{1}^{\prime}\right)+\mathbf{P B}\left(\hat{y}_{2}^{\prime}, \hat{y}_{2}^{\prime}\right) \\
& -t \mathbf{P}\left\{\mathbf{B}\left((\mathbf{I}-\mathbf{P}) \mathbf{B}\left(\hat{y}_{1}^{\prime}, \hat{y}_{3}^{\prime}\right), \hat{y}_{1}^{\prime}\right)+\mathbf{B}\left(\hat{y}_{1}^{\prime},-(\mathbf{I}-\mathbf{P}) \mathbf{B}\left(\hat{y}_{1}^{\prime}, \hat{y}_{3}^{\prime}\right)\right)\right\} \\
& +t \mathbf{P}\left\{\mathbf{B}\left(-(\mathbf{I}-\mathbf{P}) \mathbf{B}\left(\hat{y}_{2}^{\prime}, \hat{y}_{3}^{\prime}\right), \hat{y}_{2}^{\prime}\right)+\mathbf{B}\left(\hat{y}_{2}^{\prime},-(\mathbf{I}-\mathbf{P}) \mathbf{B}\left(\hat{y}_{2}^{\prime}, \hat{y}_{3}^{\prime}\right)\right)\right\} .
\end{aligned}
$$

Theorem 1. Let $\hat{\mathbf{y}}^{\prime}=\left(\hat{y}_{1}^{\prime}, \hat{y}_{2}^{\prime}, \hat{y}_{3}^{\prime}\right)^{T}$ and $\boldsymbol{\Gamma}=\left(\Gamma_{1}, \Gamma_{2}, \Gamma_{3}\right)^{T}$ where $\Gamma_{1}=(\mathbf{I}-$ $\mathbf{P}) \mathbf{B}\left(\hat{y}_{1}^{\prime}, \hat{y}_{3}^{\prime}\right), \Gamma_{2}=-(\mathbf{I}-\mathbf{P}) \mathbf{B}\left(\hat{y}_{2}^{\prime}, \hat{y}_{3}^{\prime}\right), \Gamma_{3}=-(\mathbf{I}-\mathbf{P}) \mathbf{B}\left(\hat{y}_{1}^{\prime}, \hat{y}_{1}^{\prime}\right)+(\mathbf{I}-\mathbf{P}) \mathbf{B}\left(\hat{y}_{2}^{\prime}, \hat{y}_{2}^{\prime}\right)$, then

$$
\frac{1}{2} \frac{d}{d t}\left\|\hat{\mathbf{y}}^{\prime}\right\|_{L_{2}(\Omega)}^{2}=-t\|\boldsymbol{\Gamma}\|_{L_{2}(\Omega)}^{2},
$$

Proof. For simplicity, we use $(\cdot, \cdot)$ to denote the inner product and $\|\cdot\|$ to 
denote the $L_{2}$ norm on the random space. From (C.3), we obtain

$$
\begin{aligned}
\frac{1}{2} \frac{d}{d t}\left\|\hat{y}_{1}^{\prime}\right\|^{2}= & \left(\mathbf{B}\left(\hat{y}_{1}^{\prime}, \hat{y}_{3}^{\prime}\right), \hat{y}_{1}^{\prime}\right)+t\left(\mathbf{B}\left(\Gamma_{1}, \hat{y}_{3}^{\prime}\right), \hat{y}_{1}^{\prime}\right)+t\left(\mathbf{B}\left(\hat{y}_{1}^{\prime}, \Gamma_{3}\right), \hat{y}_{1}^{\prime}\right), \\
\frac{1}{2} \frac{d}{d t}\left\|\hat{y}_{2}^{\prime}\right\|^{2}= & -\left(\mathbf{B}\left(\hat{y}_{2}^{\prime}, \hat{y}_{3}^{\prime}\right), \hat{y}_{2}^{\prime}\right)-t\left(\mathbf{B}\left(\Gamma_{2}, \hat{y}_{3}^{\prime}\right), \hat{y}_{2}^{\prime}\right)-t\left(\mathbf{B}\left(\hat{y}_{2}^{\prime}, \Gamma_{3}\right), \hat{y}_{2}^{\prime}\right), \\
\frac{1}{2} \frac{d}{d t}\left\|\hat{y}_{3}^{\prime}\right\|^{2}= & \left(-\mathbf{B}\left(\hat{y}_{1}^{\prime}, \hat{y}_{1}^{\prime}\right)+\mathbf{B}\left(\hat{y}_{2}^{\prime}, \hat{y}_{2}^{\prime}\right), \hat{y}_{3}^{\prime}\right) \\
& -t\left(\mathbf{B}\left(\Gamma_{1}, \hat{y}_{1}^{\prime}\right)+\mathbf{B}\left(\hat{y}_{1}^{\prime}, \Gamma_{1}\right), \hat{y}_{3}^{\prime}\right)+t\left(\mathbf{B}\left(\Gamma_{2}, \hat{y}_{2}^{\prime}\right)+\mathbf{B}\left(\hat{y}_{2}^{\prime}, \Gamma_{2}\right), \hat{y}_{3}^{\prime}\right) .
\end{aligned}
$$

First, we claim that

$$
(\mathbf{B}(f, g), h)=(\mathbf{B}(f, h), g)=(\mathbf{B}(g, h), f) .
$$

To show (C.6), we assume $f=\sum_{\mathbf{i}} f_{\mathbf{i}} \Phi_{\mathbf{i}}, g=\sum_{\mathbf{i}} g_{\mathbf{i}} \Phi_{\mathbf{i}}, h=\sum_{\mathbf{i}} h_{\mathbf{i}} \Phi_{\mathbf{i}}$. We find

$$
\begin{aligned}
(\mathbf{B}(f, g), h) & =\left(\mathbf{B}\left(\sum_{\mathbf{i}} f_{\mathbf{i}} \Phi_{\mathbf{i}}, \sum_{\mathbf{j}} g_{\mathbf{j}} \Phi_{\mathbf{j}}\right), \sum_{\mathbf{k}} h_{\mathbf{k}} \Phi_{\mathbf{k}}\right) \\
& =\sum_{\mathbf{i}} \sum_{\mathbf{j}} \sum_{\mathbf{k}} f_{\mathbf{i}} g_{\mathbf{j}} h_{\mathbf{k}} \int_{\Omega} \Phi_{\mathbf{i}} \Phi_{\mathbf{j}} \Phi_{\mathbf{k}} d \mathcal{P} .
\end{aligned}
$$

Obviously, it can be verified that

$$
\begin{aligned}
& (\mathbf{B}(f, h), g)=\sum_{\mathbf{i}} \sum_{\mathbf{j}} \sum_{\mathbf{k}} f_{\mathbf{i}} g_{\mathbf{j}} h_{\mathbf{k}} \int_{\Omega} \Phi_{\mathbf{i}} \Phi_{\mathbf{j}} \Phi_{\mathbf{k}} d \mathcal{P}, \\
& (\mathbf{B}(g, h), f)=\sum_{\mathbf{i}} \sum_{\mathbf{j}} \sum_{\mathbf{k}} f_{\mathbf{i}} g_{\mathbf{j}} h_{\mathbf{k}} \int_{\Omega} \Phi_{\mathbf{i}} \Phi_{\mathbf{j}} \Phi_{\mathbf{k}} d \mathcal{P} .
\end{aligned}
$$

Consequently, (C.6) is satisfied. Given the fact that $\mathbf{P} \perp(\mathbf{I}-\mathbf{P})$, and (C.6), we 
have

$$
\begin{aligned}
& \frac{1}{2} \frac{d}{d t}\left\|\hat{\mathbf{y}}^{\prime}\right\|^{2}=t\left(\mathbf{B}\left(\hat{y}_{1}^{\prime}, \Gamma_{3}\right), \hat{y}_{1}^{\prime}\right)-t\left(\mathbf{B}\left(\hat{y}_{2}^{\prime}, \Gamma_{3}\right), \hat{y}_{2}^{\prime}\right) \\
& -t\left(\mathbf{B}\left(\Gamma_{1}, \hat{y}_{1}^{\prime}\right), \hat{y}_{3}^{\prime}\right)+t\left(\mathbf{B}\left(\hat{y}_{2}^{\prime}, \Gamma_{2}\right), \hat{y}_{3}^{\prime}\right) \\
& =t\left(\mathbf{B}\left(\hat{y}_{1}^{\prime},-(\mathbf{I}-\mathbf{P}) \mathbf{B}\left(\hat{y}_{1}^{\prime}, \hat{y}_{1}^{\prime}\right)+(\mathbf{I}-\mathbf{P}) \mathbf{B}\left(\hat{y}_{2}^{\prime}, \hat{y}_{2}^{\prime}\right)\right), \hat{y}_{1}^{\prime}\right) \\
& -t\left(\mathbf{B}\left(\hat{y}_{2}^{\prime},-(\mathbf{I}-\mathbf{P}) \mathbf{B}\left(\hat{y}_{1}^{\prime}, \hat{y}_{1}^{\prime}\right)+(\mathbf{I}-\mathbf{P}) \mathbf{B}\left(\hat{y}_{2}^{\prime}, \hat{y}_{2}^{\prime}\right)\right), \hat{y}_{2}^{\prime}\right) \\
& -t\left(\mathbf{B}\left(\Gamma_{1}, \hat{y}_{1}^{\prime}\right), \hat{y}_{3}^{\prime}\right)+t\left(\mathbf{B}\left(\hat{y}_{2}^{\prime}, \Gamma_{2}\right), \hat{y}_{3}^{\prime}\right) \\
& =-t\left(\mathbf{B}\left(\hat{y}_{1}^{\prime}, \hat{y}_{1}^{\prime}\right),(\mathbf{I}-\mathbf{P}) \mathbf{B}\left(\hat{y}_{1}^{\prime}, \hat{y}_{1}^{\prime}\right)\right)+t\left(\mathbf{B}\left(\hat{y}_{2}^{\prime}, \hat{y}_{2}^{\prime}\right),(\mathbf{I}-\mathbf{P}) \mathbf{B}\left(\hat{y}_{1}^{\prime}, \hat{y}_{1}^{\prime}\right)\right) \\
& +t\left(\mathbf{B}\left(\hat{y}_{2}^{\prime} \hat{y}_{2}^{\prime}\right),(\mathbf{I}-\mathbf{P}) \mathbf{B}\left(\hat{y}_{1}^{\prime}, \hat{y}_{1}^{\prime}\right)\right)-t\left(\mathbf{B}\left(\hat{y}_{2}^{\prime}, \hat{y}_{2}^{\prime}\right),(\mathbf{I}-\mathbf{P}) \mathbf{B}\left(\hat{y}_{2}^{\prime}, \hat{y}_{2}^{\prime}\right)\right) \\
& -t\left(\mathbf{B}\left(\Gamma_{1}, \hat{y}_{1}^{\prime}\right), y_{3}^{\prime}\right)+t\left(\mathbf{B}\left(\hat{y}_{2}^{\prime}, \Gamma_{2}\right), \hat{y}_{3}^{\prime}\right) \\
& =-t\left\|(\mathbf{I}-\mathbf{P}) \mathbf{B}\left(\hat{y}_{1}^{\prime}, \hat{y}_{1}^{\prime}\right)\right\|^{2}-t\left\|(\mathbf{I}-\mathbf{P}) \mathbf{B}\left(\hat{y}_{2}^{\prime}, \hat{y}_{2}^{\prime}\right)\right\|^{2} \\
& +2 t\left((\mathbf{I}-\mathbf{P}) \mathbf{B}\left(\hat{y}_{1}^{\prime}, \hat{y}_{1}^{\prime}\right),(\mathbf{I}-\mathbf{P}) \mathbf{B}\left(\hat{y}_{2}^{\prime}, \hat{y}_{2}^{\prime}\right)\right) \\
& -t\left(\mathbf{B}\left(\hat{y}_{1}^{\prime}, \hat{y}_{3}^{\prime}\right), \Gamma_{1}\right)+t\left(\mathbf{B}\left(\hat{y}_{2}^{\prime}, \hat{y}_{3}^{\prime}\right), \Gamma_{2}\right) \\
& =-t\left\|(\mathbf{I}-\mathbf{P}) \mathbf{B}\left(\hat{y}_{1}^{\prime}, \hat{y}_{1}^{\prime}\right)-(\mathbf{I}-\mathbf{P}) \mathbf{B}\left(\hat{y}_{2}^{\prime}, \hat{y}_{2}^{\prime}\right)\right\|^{2} \\
& -t\left(\mathbf{B}\left(\hat{y}_{1}^{\prime}, \hat{y}_{3}^{\prime}\right),(\mathbf{I}-\mathbf{P}) \mathbf{B}\left(\hat{y}_{1}^{\prime}, \hat{y}_{3}^{\prime}\right)\right)-t\left(-\mathbf{B}\left(\hat{y}_{2}^{\prime}, \hat{y}_{3}^{\prime}\right),-(\mathbf{I}-\mathbf{P}) \mathbf{B}\left(\hat{y}_{2}^{\prime}, \hat{y}_{3}^{\prime}\right)\right) \\
& =-t\left\|\Gamma_{3}\right\|^{2}-t\left\|\Gamma_{1}\right\|^{2}-t\left\|\Gamma_{2}\right\|^{2} \text {. }
\end{aligned}
$$

Theorem 1 shows that in Kraichnan-Orszag system the rate of the change of the $L_{2}$ can be computed by the contribution of t- model term.

With the same notations as before, we have the following theorem which characterizes the error of the $t$-model system.

Theorem 2. Let $\mathbf{y}=\left(y_{1}, y_{2}, y_{3}\right)^{T}$, and $\mathbf{P y}=\left(\mathbf{P} y_{1}, \mathbf{P} y_{2}, \mathbf{P} y_{3}\right)^{T}$, where $y_{1}, y_{2}, y_{3}$ satisfy (17). Then, there exist constants $A, B$, and $C$ such that

$$
\begin{aligned}
\frac{1}{2} \frac{d}{d t}\left\|\hat{\mathbf{y}}^{\prime}-\mathbf{P y}\right\|^{2} \leq & (A+t B)\left\|\hat{\mathbf{y}}^{\prime}-\mathbf{P y}\right\|^{2}+C\|(\mathbf{I}-\mathbf{P}) \mathbf{y}\|^{2} \\
& +5 t\left\|(\mathbf{I}-\mathbf{P}) \mathbf{B}\left(\mathbf{P} y_{1}, \mathbf{P} y_{3}\right)\right\|^{2}+5 t\left\|(\mathbf{I}-\mathbf{P}) \mathbf{B}\left(\mathbf{P} y_{2}, \mathbf{P} y_{3}\right)\right\|^{2} \\
& +t\left\|(\mathbf{I}-\mathbf{P}) \mathbf{B}\left(\mathbf{P} y_{1}, \mathbf{P} y_{1}\right)\right\|^{2}+t\left\|(\mathbf{I}-\mathbf{P}) \mathbf{B}\left(\mathbf{P} y_{2}, \mathbf{P} y_{2}\right)\right\|^{2}
\end{aligned}
$$

Proof. The left side of (C.7) can be expressed as

$$
\begin{aligned}
\frac{1}{2} \frac{d}{d t}\left\|\hat{\mathbf{y}}^{\prime}-\mathbf{P y}\right\|^{2} & =\frac{1}{2} \frac{d}{d t}\left(\hat{\mathbf{y}}^{\prime}-\mathbf{P y}, \hat{\mathbf{y}}^{\prime}-\mathbf{P y}\right) \\
& =\frac{1}{2} \sum_{i=1}^{3}\left(\hat{y}_{i}^{\prime}-\mathbf{P} y_{i}, \frac{d}{d t}\left(\hat{y}_{i}^{\prime}-\mathbf{P} y_{i}\right)\right) .
\end{aligned}
$$


For $\hat{y}_{1}^{\prime}-\mathbf{P} y_{1}$,

$$
\begin{aligned}
\left(\hat{y}_{1}^{\prime}-\mathbf{P} y_{1}, \frac{d}{d t}\left(\hat{y}_{1}^{\prime}-\mathbf{P} y_{1}\right)\right) & =\left(\hat{y}_{1}^{\prime}-\mathbf{P} y_{1}, \mathbf{P B}\left(\hat{y}_{1}^{\prime}, \hat{y}_{3}^{\prime}\right)-\mathbf{P B}\left(y_{1}, y_{3}\right)\right) \\
& +\left(\hat{y}_{1}^{\prime}-\mathbf{P} y_{1}, t \mathbf{P}\left\{\mathbf{B}\left(\Gamma_{1}, \hat{y}_{3}^{\prime}\right)+\mathbf{B}\left(\hat{y}_{1}^{\prime}, \Gamma_{3}\right)\right\}\right)
\end{aligned}
$$

where $\hat{\mathbf{y}}^{\prime}$ satisfies (C.3) and Py satisfies (C.2). Let $I_{1}=\left(\hat{y}_{1}^{\prime}-\mathbf{P} y_{1}, \mathbf{P B}\left(\hat{y}_{1}^{\prime}, \hat{y}_{3}^{\prime}\right)-\right.$ $\left.\mathbf{P B}\left(y_{1}, y_{3}\right)\right), I_{2}=\left(\hat{y}_{1}^{\prime}, t \mathbf{P}\left\{\mathbf{B}\left(\Gamma_{1}, \hat{y}_{3}^{\prime}\right)+\mathbf{B}\left(\hat{y}_{1}^{\prime}, \Gamma_{3}\right)\right\}\right)$ and $I_{3}=-\left(\mathbf{P} y_{1}, t \mathbf{P}\left\{\mathbf{B}\left(\Gamma_{1}, \hat{y}_{3}^{\prime}\right)+\right.\right.$ $\left.\left.\mathbf{B}\left(\hat{y}_{1}^{\prime}, \Gamma_{3}\right)\right\}\right)$. Since $\mathbf{P}$ is self-adjoint and $\mathbf{B}$ is continuous, it follows that

$$
\begin{aligned}
I_{1} & =\left(\hat{y}_{1}^{\prime}-\mathbf{P} y_{1}, \mathbf{B}\left(\hat{y}_{1}^{\prime}, \hat{y}_{3}^{\prime}\right)-\mathbf{B}\left(y_{1}, y_{3}\right)\right) \\
& \leq\left\|\hat{y}_{1}^{\prime}-\mathbf{P} y_{1}\right\|\left\|\mathbf{B}\left(\hat{y}_{1}^{\prime}, \hat{y}_{3}^{\prime}\right)-\mathbf{B}\left(y_{1}, y_{3}\right)\right\| \\
& \leq\|\hat{\mathbf{y}}-\mathbf{P y}\| C_{1}\|\hat{\mathbf{y}}-\mathbf{y}\| \\
& \leq C_{1}\left\|\hat{\mathbf{y}}^{\prime}-\mathbf{P y}\right\|\left(\left\|\hat{\mathbf{y}}^{\prime}-\mathbf{P y}\right\|+\|(\mathbf{I}-\mathbf{P}) \mathbf{y}\|\right) \\
& =C_{1}\left\|\hat{\mathbf{y}}^{\prime}-\mathbf{P y}\right\|^{2}+C_{1}\left\|\hat{\mathbf{y}}^{\prime}-\mathbf{P y}\right\|\|(\mathbf{I}-\mathbf{P}) \mathbf{y}\|,
\end{aligned}
$$

where $C_{1}$ is some constant. Also, for $I_{3}$ we have

$$
\begin{aligned}
I_{3}= & -t\left(\mathbf{P} y_{1}, \mathbf{P}\left\{\mathbf{B}\left(\Gamma_{1}, \hat{y}_{3}^{\prime}\right)+\mathbf{B}\left(\hat{y}_{1}^{\prime}, \Gamma_{3}\right)\right\}\right) \\
= & -t\left(\mathbf{P} y_{1}, \mathbf{P B}\left(\Gamma_{1}, \hat{y}_{3}^{\prime}-\mathbf{P} y_{3}\right)\right)-t\left(\mathbf{P} y_{1}, \mathbf{P B}\left(\Gamma_{1}, \mathbf{P} y_{3}\right)\right) \\
& -t\left(\mathbf{P} y_{1}, \mathbf{P B}\left(\Gamma_{3}, \hat{y}_{1}^{\prime}-\mathbf{P} y_{1}\right)\right)-t\left(\mathbf{P} y_{1}, \mathbf{P B}\left(\Gamma_{3}, \mathbf{P} y_{1}\right)\right) \\
= & -t\left(\mathbf{P} y_{1}^{\prime}\left(\hat{y}_{3}^{\prime}-\mathbf{P} y_{3}\right), \Gamma_{1}\right)-\left(\mathbf{P} y_{1} \mathbf{P} y_{3}, \Gamma_{1}\right) \\
& -t\left(\mathbf{P} y_{1}^{\prime}\left(\hat{y}_{1}^{\prime}-\mathbf{P} y_{1}\right), \Gamma_{3}\right)-\left(\mathbf{P} y_{1} \mathbf{P} y_{1}, \Gamma_{3}\right) \\
= & -t\left(\mathbf{P} y_{1}^{\prime}\left(\hat{y}_{3}^{\prime}-\mathbf{P} y_{3}\right), \Gamma_{1}\right)-t\left((\mathbf{I}-\mathbf{P}) \mathbf{P} y_{1} \mathbf{P} y_{3}, \Gamma_{1}\right) \\
& -t\left(\mathbf{P} y_{1}\left(\hat{y}_{1}^{\prime}-\mathbf{P} y_{1}\right), \Gamma_{3}\right)-t\left((\mathbf{I}-\mathbf{P})\left(\mathbf{P} y_{1}\right)^{2}, \Gamma_{3}\right) \\
\leq & t\left\|\mathbf{P} y_{1}\left(\hat{y}_{3}^{\prime}-\mathbf{P} y_{3}\right)\right\|\left\|\Gamma_{1}\right\|+t\left\|(\mathbf{I}-\mathbf{P})\left(\mathbf{P} y_{1} \mathbf{P} y_{3}\right)\right\|\left\|\Gamma_{1}\right\| \\
& +t\left\|\mathbf{P} y_{1}\left(\hat{y}_{1}^{\prime}-\mathbf{P} y_{1}\right)\right\|\left\|\Gamma_{3}\right\|+t\left\|(\mathbf{I}-\mathbf{P})\left(\mathbf{P} y_{1}\right)^{2}\right\|\left\|\Gamma_{3}\right\| \\
\leq & t\left\|\mathbf{P} y_{1}\right\|_{L^{\infty}}^{2}\left\|\hat{y}_{3}^{\prime}-\mathbf{P} y_{3}\right\|^{2}+\frac{t}{4}\left\|\Gamma_{1}\right\|^{2} \\
& +t\left\|(\mathbf{I}-\mathbf{P})\left(\mathbf{P} y_{1} \mathbf{P} y_{3}\right)\right\|^{2}+\frac{t}{4}\left\|\Gamma_{1}\right\|^{2} \\
& +t\left\|\mathbf{P} y_{1}\right\|_{L^{\infty}}^{2}\left\|\hat{y}_{1}^{\prime}-\mathbf{P} y_{1}\right\|^{2}+\frac{t}{4}\left\|\Gamma_{3}\right\|^{2} \\
& +t\left\|(\mathbf{I}-\mathbf{P})\left(\mathbf{P} y_{1}\right)^{2}\right\|^{2}+\frac{t}{4}\left\|\Gamma_{3}\right\|^{2} \\
\leq & t\left\|\mathbf{P} y_{1}\right\|_{L^{\infty}}^{2}\left\|\hat{\mathbf{y}}^{\prime}-\mathbf{P y}\right\|^{2}+t\left\|(\mathbf{I}-\mathbf{P})\left(\mathbf{P} y_{1} \mathbf{P} y_{3}\right)\right\|^{2}+\frac{t}{2}\left\|\Gamma_{1}\right\|^{2}
\end{aligned}
$$




$$
+t\left\|(\mathbf{I}-\mathbf{P})\left(\mathbf{P} y_{1}\right)^{2}\right\|^{2}+\frac{t}{2}\left\|\Gamma_{3}\right\|^{2} .
$$

For $\hat{y}_{2}^{\prime}-\mathbf{P} y_{2}$, and $\hat{y}_{3}^{\prime}-\mathbf{P} y_{3}$, we have

$$
\begin{aligned}
\left(\hat{y}_{2}^{\prime}-\mathbf{P} y_{2}, \frac{d}{d t}\left(\hat{y}_{2}^{\prime}-\mathbf{P} y_{2}\right)\right)= & \left(\hat{y}_{2}^{\prime}-\mathbf{P} y_{2},-\mathbf{P B}\left(\hat{y}_{2}^{\prime}, \hat{y}_{3}^{\prime}\right)+\mathbf{P B}\left(y_{2}, y_{3}\right)\right) \\
& -\left(\hat{y}_{2}^{\prime}, t \mathbf{P}\left\{\mathbf{B}\left(\Gamma_{2}, \hat{y}_{3}^{\prime}\right)+\mathbf{B}\left(\hat{y}_{2}^{\prime}, \Gamma_{3}\right)\right\}\right) \\
& +\left(\mathbf{P} y_{2}, t \mathbf{P}\left\{\mathbf{B}\left(\Gamma_{2}, \hat{y}_{3}^{\prime}\right)+\mathbf{B}\left(\hat{y}_{2}^{\prime}, \Gamma_{3}\right)\right\}\right) \\
\triangleq & J_{1}+J_{2}+J_{3}, \\
\left(\hat{y}_{3}^{\prime}-\mathbf{P} y_{3}, \frac{d}{d t}\left(\hat{y}_{3}^{\prime}-\mathbf{P} y_{3}\right)\right)= & \hat{y}_{3}^{\prime}-\mathbf{P} y_{3},-\mathbf{P B}\left(\hat{y}_{1}^{\prime}, \hat{y}_{1}^{\prime}\right)+\mathbf{P B}\left(\hat{y}_{2}^{\prime}, \hat{y}_{2}^{\prime}\right) \\
& \left.+\mathbf{P B}\left(y_{1}, y_{1}\right)-\mathbf{P B}\left(\hat{y}_{2}^{\prime}, \hat{y}_{2}^{\prime}\right)\right) \\
& -2\left(\hat{y}_{1}^{\prime}, t \mathbf{P}\left\{\mathbf{B}\left(\Gamma_{1}, \hat{y}_{1}^{\prime}\right)\right\}\right)+2\left(\hat{y}_{2}^{\prime}, t \mathbf{P}\left\{\mathbf{B}\left(\Gamma_{2}, \hat{y}_{2}^{\prime}\right)\right\}\right) \\
& +2\left(\mathbf{P} y_{1}, t \mathbf{P}\left\{\mathbf{B}\left(\Gamma_{1}, \hat{y}_{1}^{\prime}\right)\right\}\right)-2\left(\mathbf{P} y_{2}, t \mathbf{P}\left\{\mathbf{B}\left(\Gamma_{2}, \hat{y}_{2}^{\prime}\right)\right\}\right) \\
\triangleq & K_{1}+K_{2}+K_{3} .
\end{aligned}
$$

Following the same steps as in (C.10) and (C.11), we obtain

$$
\begin{aligned}
J_{1} \leq & C_{1}\left\|\hat{\mathbf{y}}^{\prime}-\mathbf{P y}\right\|^{2}+C_{1}\left\|\hat{\mathbf{y}}^{\prime}-\mathbf{P y}\right\|\|(\mathbf{I}-\mathbf{P}) \mathbf{y}\| \\
J_{3} \leq & t\left\|\mathbf{P} y_{2}\right\|_{L^{\infty}}^{2}\|\hat{\mathbf{y}}-\mathbf{P y}\|^{2}+t\left\|(\mathbf{I}-\mathbf{P})\left(\mathbf{P} y_{2} \mathbf{P} y_{3}\right)\right\|^{2}+\frac{t}{2}\left\|\Gamma_{2}\right\|^{2} \\
& +t\left\|(\mathbf{I}-\mathbf{P})\left(\mathbf{P} y_{2}\right)^{2}\right\|^{2}+\frac{t}{2}\left\|\Gamma_{3}\right\|^{2} ; \\
K_{1} \leq & C_{2}\left\|\hat{\mathbf{y}}^{\prime}-\mathbf{P y}\right\|^{2}+C_{2}\left\|\hat{\mathbf{y}}^{\prime}-\mathbf{P y}\right\|\|(\mathbf{I}-\mathbf{P}) \mathbf{y}\|, \text { for some constant } C_{2} \\
K_{3} \leq & 4 t\left\|\mathbf{P} y_{3}\right\|_{L^{\infty}}^{2}\|\hat{\mathbf{y}}-\mathbf{P y}\|^{2}+4 t\left\|(\mathbf{I}-\mathbf{P})\left(\mathbf{P} y_{3} \mathbf{P} y_{1}\right)\right\|^{2} \\
& +4 t\left\|(\mathbf{I}-\mathbf{P})\left(\mathbf{P} y_{3} \mathbf{P} y_{2}\right)\right\|^{2}+\frac{t}{2}\left\|\Gamma_{1}\right\|^{2}+\frac{t}{2}\left\|\Gamma_{2}\right\|^{2} .
\end{aligned}
$$

Finally, it is easy to verify that $I_{2}+J_{2}+K_{2}=\frac{1}{2} \frac{d}{d t}\left\|\hat{\mathbf{y}}^{\prime}\right\|^{2}=-t\|\boldsymbol{\Gamma}\|^{2}$. Putting everything together we see that there exist constants $C_{3}, C_{4}, A, B$ and $C$ such that

$$
\begin{aligned}
\frac{1}{2} \frac{d}{d t}\left\|\hat{\mathbf{y}}^{\prime}-\mathbf{P y}\right\|^{2} \leq & C_{3}\left\|\hat{\mathbf{y}}^{\prime}-\mathbf{P y}\right\|^{2}+C_{4}\left\|\hat{\mathbf{y}}^{\prime}-\mathbf{P y}\right\|\|(\mathbf{I}-\mathbf{P}) \mathbf{y}\| \\
& +t \max \left(\left\|\mathbf{P} y_{1}\right\|_{L^{\infty}}^{2},\left\|\mathbf{P} y_{2}\right\|_{L^{\infty}}^{2}, 4\left\|\mathbf{P} y_{3}\right\|_{L^{\infty}}^{2}\right)\|\hat{\mathbf{y}}-\mathbf{P y}\|^{2} \\
& +5 t\left\|(\mathbf{I}-\mathbf{P})\left(\mathbf{P} y_{1} \mathbf{P} y_{3}\right)\right\|^{2}+5 t\left\|(\mathbf{I}-\mathbf{P})\left(\mathbf{P} y_{2} \mathbf{P} y_{3}\right)\right\|^{2} \\
& +t\left\|(\mathbf{I}-\mathbf{P})\left(\mathbf{P} y_{1}\right)^{2}\right\|^{2}+t\left\|(\mathbf{I}-\mathbf{P})\left(\mathbf{P} y_{2}\right)^{2}\right\|^{2} \\
\leq & (A+t B)\left\|\hat{\mathbf{y}}^{\prime}-\mathbf{P y}\right\|^{2}+C\|(\mathbf{I}-\mathbf{P}) \mathbf{y}\|^{2} \\
& +5 t\left\|(\mathbf{I}-\mathbf{P})\left(\mathbf{P} y_{1} \mathbf{P} y_{3}\right)\right\|^{2}+5 t\left\|(\mathbf{I}-\mathbf{P})\left(\mathbf{P} y_{2} \mathbf{P} y_{3}\right)\right\|^{2} \\
& +t\left\|(\mathbf{I}-\mathbf{P})\left(\mathbf{P} y_{1}\right)^{2}\right\|^{2}+t\left\|(\mathbf{I}-\mathbf{P})\left(\mathbf{P} y_{2}\right)^{2}\right\|^{2}
\end{aligned}
$$


From (C.13) we can see that the contribution of the $t$-model term to the error of $t$-model approximation is expressed as

$$
\begin{aligned}
& 5 t\left\|(\mathbf{I}-\mathbf{P}) \mathbf{B}\left(\mathbf{P} y_{1}, \mathbf{P} y_{3}\right)\right\|^{2}+5 t\left\|(\mathbf{I}-\mathbf{P}) \mathbf{B}\left(\mathbf{P} y_{2}, \mathbf{P} y_{3}\right)\right\|^{2} \\
& +t\left\|(\mathbf{I}-\mathbf{P}) \mathbf{B}\left(\mathbf{P} y_{1}, \mathbf{P} y_{1}\right)\right\|^{2}+t\left\|(\mathbf{I}-\mathbf{P}) \mathbf{B}\left(\mathbf{P} y_{2}, \mathbf{P} y_{2}\right)\right\|^{2} .
\end{aligned}
$$

Compared with

$$
\begin{aligned}
\frac{1}{2}\left|\frac{d}{d t}\left\|\hat{\mathbf{y}}^{\prime}\right\|^{2}\right| \leq & t\left(\left\|(\mathbf{I}-\mathbf{P}) \mathbf{B}\left(\hat{y}_{1}^{\prime}, \hat{y}_{3}^{\prime}\right)\right\|^{2}+\left\|(\mathbf{I}-\mathbf{P}) \mathbf{B}\left(\hat{y}_{2}^{\prime}, \hat{y}_{3}^{\prime}\right)\right\|^{2}\right. \\
& \left.+2\left\|(\mathbf{I}-\mathbf{P}) \mathbf{B}\left(\hat{y}_{1}^{\prime}, \hat{y}_{1}^{\prime}\right)\right\|^{2}+2\left\|(\mathbf{I}-\mathbf{P}) \mathbf{B}\left(\hat{y}_{2}^{\prime}, \hat{y}_{2}^{\prime}\right)\right\|^{2}\right),
\end{aligned}
$$

we can conclude that $\frac{1}{2}\left|\frac{d}{d t}\left\|\hat{\mathbf{y}}^{\prime}\right\|^{2}\right|$ is a good indicator of the rate of change of the error due to the $t$-model term. Meanwhile the error generated by the $t$ model term signifies the energy moving from the resolved modes to the unresolved modes of the full system. Thus the error can be controlled by controlling $\left|\frac{d}{d t}\left\|\hat{\mathbf{y}}^{\prime}\right\|^{2}\right|$. In particular, $\left|\frac{d}{d t}\left\|\hat{\mathbf{y}}^{\prime}\right\|^{2}\right| \leq T O L_{1}$ provides a good criterion for mesh refinement. 